\title{
Lipoproteins and lipoprotein mimetics for imaging and drug delivery
}

C. Shad Thaxton, ${ }^{1,2,3,4}$ Jonathan S. Rink ${ }^{1,2}$ Pratap C. Naha, ${ }^{5}$ David P. Cormode ${ }^{5,6,7 *}$

Department of Urology ${ }^{1}$, Simpson Querrey Institute for Bionanotechnology ${ }^{2}$,

International Institute for Nanotechnology ${ }^{3}$, Robert H. Lurie Comprehensive Cancer Center ${ }^{4}$ Northwestern University, Chicago, IL, USA

Departments of Radiology, ${ }^{5}$ Bioengineering, ${ }^{6}$ Cardiology, ${ }^{7}$ University of Pennsylvania, 3400 Spruce St, 1 Silverstein, Philadelphia, PA 19104, USA, Tel: 215-615-4656, Fax: 240-368-8096 david.cormode@uphs.upenn.edu

* Corresponding Author 


\begin{abstract}
Lipoproteins are a set of natural nanoparticles whose main role is the transport of fats within the body. While much work has been done to develop synthetic nanocarriers to deliver drugs or contrast media, natural nanoparticles such as lipoproteins represent appealing alternatives. Lipoproteins are biocompatible, biodegradable, non-immunogenic and are naturally targeted to some disease sites. Lipoproteins can be modified to act as contrast agents in many ways, such as by insertion of gold cores to provide contrast for computed tomography. They can be loaded with drugs, nucleic acids, photosensitizers or boron to act as therapeutics. Attachment of ligands can re-route lipoproteins to new targets. These attributes render lipoproteins attractive and versatile delivery vehicles. In this review we will provide background on lipoproteins, then survey their roles as contrast agents, in drug and nucleic acid delivery, as well as in photodynamic therapy and boron neutron capture therapy.
\end{abstract}

Keywords: high-density lipoprotein, low-density lipoprotein, drug delivery, siRNA, MRI, fluorescence imaging, computed tomography, photodynamic therapy, boron neutron capture therapy 


\section{Introduction}

There is a tremendous need for safe and effective delivery vehicles in both the fields of imaging and therapeutics. Medical imaging benefits from the use of contrast agents to allow additional and improved diagnoses. In particular, the field of molecular imaging has recently emerged, where precise details of pathologies, such as levels of certain cells or disease phenotype, can be determined via the use of contrast agents.[1] The agents used for molecular imaging are frequently targeted via some kind of ligand such as an antibody, protein, peptide or aptamer. For many imaging techniques, such as magnetic resonance imaging (MRI), computed tomography (CT), photoacoustics, fluorescence imaging, surface enhanced Raman spectroscopy (SERS) imaging and others, nanoparticle based agents have been shown to be particularly effective as contrast agents.[1-5] Nanoparticles offer advantages such as high payloads, contrast that is not available with small molecules or greatly enhanced contrast, ease of integration of multiple properties, long circulation times and efficient targeting.[6]

In the case of drug delivery, nanoparticle based agents are starting to form a significant part of our array of disease treatments.[7] For example, Abraxane is a nanoparticle formed from albumin with a payload of paclitaxel, approved for treatment of breast, lung and pancreatic cancers, among others.[8] The sales of this nanoparticle drug alone are estimated to be $\$ 1$ billion for 2015.[9] Nanoparticle delivery vehicles offer solutions to a number of problems, such as rapid clearance of injected or ingested drugs, poor bioavailability, low drug solubility, metabolism in circulation, lack of access to target sites and so forth. Therefore, there are enormous efforts in both academia and industry to develop nanoparticle-based therapeutic systems. 
Due to the tremendous interest in nanoparticles as delivery vehicles for both contrast media and therapeutics, numerous synthetic nanoparticle platforms have been explored for these purposes. These include lipid-based systems such as micelles, liposomes and emulsions.[10-13] The first nanoparticle to be FDA-approved for medical use was Doxil, a liposomal formulation of Doxorubicin.[7] Other synthetic nanoparticle based platforms that have been proposed include polymer platforms such as PLGA (poly lactic-co-glycolic acid),[14, 15] polymer-lipid hybrid nanoparticles,[16, 17] dendrimers,[18] graphene,[19, 20] gold nanoparticles,[4, 21] iron oxides,[22-25] and silica,[26, 27] among others. While many of the platforms have met with success, there are general concerns over synthetic nanoparticles, such as the potential for immunogenicity, biocompatibility, excretion and degradation. These concerns have inspired investigations of natural nanoparticles as delivery platforms, since natural nanoparticles would be likely to be non-immunogenic, biocompatible and degradable.[28] Lipoproteins,[29-32] viruses,[33, 34] ferritin[35-37] and, more recently, vault proteins[38] have been considered as natural nanoparticle delivery candidates.

Amongst these platforms, lipoproteins have been the focus of much of the research. This is because they are endogenous, they can deliver a range of payloads (contrast media, drugs and nucleic acids),[28-32] they naturally accumulate at several important targets [e.g. macrophages, the low density lipoprotein (LDL) receptor, scavenger receptor type B1 (SR-B1)],[39-41] they can be re-routed to other targets,[42, 43] they can carry lipophilic drugs,[44, 45] they can be modified in numerous different methods and imaging their behavior is of fundamental importance. Lipoproteins, based on their size and surface composition, may demonstrate relatively long circulating half- 
lives, compared with non-lipoprotein nanoparticles.[46, 47] The progress to date in the use of lipoproteins to deliver contrast media or therapeutics will be the topic of this review article. We will first discuss the composition, different classes and natural roles of lipoproteins. We then consider some of the characteristics that classify synthetic nanoparticles as mimetics of lipoproteins. Following is a description of the use of lipoproteins as contrast agents for medical imaging techniques such as MRI, CT, fluorescence imaging, positron emission tomography (PET) and so on. We then review the work in drug and nucleic acid delivery, photodynamic therapy (PDT), as well as boron neutron capture therapy (BNCT). Some studies are discussed in detail in order to give the reader a better understanding of how delivery agents can be developed based on lipoproteins.

\section{Lipoproteins}

There are several classes of lipoproteins: chylomicrons (80-1200), very low density lipoprotein (VLDL; 35-80 $\mathrm{nm}$ in diameter), intermediate density lipoprotein (IDL; $27-30 \mathrm{~nm}$ in diameter), low density lipoprotein (LDL; 22-27 nm in diameter) and high density lipoprotein (HDL; 7-13 $\mathrm{nm}$ in diameter).[48] The lipoproteins are thus named due to their differing densities and are frequently separated from plasma using a density-based ultracentrifugation procedure.[49] They all have similar structures where a core composed of triglycerides and cholesterol esters is covered with a layer of phospholipids, into which is embedded amphipathic proteins termed apolipoproteins (Figure 1).[50] The lipoproteins differ in their size, lipid composition, major 
apolipoproteins, function and, of course, density. The characteristics of the lipoproteins are summarized in Table 1.

Chylomicrons are the largest lipoprotein at $80-1200 \mathrm{~nm}$ in size.[51] Dietary fats taken up in the intestines by enterocytes are packaged into chylomicrons and transferred into the lymphatic system.[52] From there they move into the blood, depositing trigylcerides in peripheral tissues via interaction with lipoprotein lipase and the remnant particles are removed by the liver, with a half-life of only a few minutes.[53] As such, their major role is the transfer of dietary fat into the body.

VLDL is formed in hepatocytes in the liver, via a complex, multi-step process.[54] It contains endogenously produced lipids. It also distributes triglycerides to the peripheral tissues via interactions with lipoprotein lipase.[55] This process forms IDL, which is hydrolysed by hepatic lipase to form LDL.[56] In the liver, LDL picks up cholesterol that is from the diet or has been synthesized in hepatocytes, with 3-hydroxy3-methylglutaryl coenzyme A reductase (HMGCR) being the rate-limiting enzyme in cholesterol synthesis.[57] LDL then transports cholesterol to the peripheral tissues.[58] Immature HDL (i.e. discoidal, lipid deficient) is formed in the peripheral tissues. HDL matures by obtaining more cholesterol and lipids from peripheral tissues via interactions between apoA-I and ATP-binding cassette A1 (ABC-A1).[59] After interactions with cholesterol ester transfer protein and endothelial lipase, HDL deposits cholesterol in the liver via the SR-BI receptor for excretion or for redistribution via LDL.[60] Lecithincholesterol acyltransferase (LCAT) also plays a role in the maturation of HDL from discoidal into its more cholesterol rich spherical forms, by converting cholesterol into cholesterol esters, which then moves into the particle core.[61] 
Beyond the major classifications of lipoproteins, there are sub-populations that are structurally, compositionally and functionally heterogeneous. For example, HDL can be divided into HDL2a, HDL2b, HDL3a, HDL3b and HDL3c by centrifugation and gel electrophoresis.[61] These sub-populations differ in size, amount of lipid and the proportions of various lipids in their composition. While the major apolipoprotein component of HDL is apoA-I, as many as 85 proteins have been identified as being associated with it.[62] Furthermore, the roles of lipoproteins continue to be revealed and are highly complex, with HDL having effects in oxidation, inflammation, hemostasis and immunity.[62]

The lipoproteins are known to play important roles in cardiovascular disease. Plasma levels of LDL-cholesterol are correlated with risk of coronary artery disease.[63] Lowering plasma levels of LDL-cholesterol has been shown to reduce the risk of coronary artery disease, and is widely done with a class of drug known as statins.[64] Statins inhibit the HMGCR enzyme, reducing cholesterol synthesis in the liver, which results in increased expression of the LDL receptor (LDLr) in the liver and greater clearance of LDL from the blood.[65, 66] More recently, antibodies against PCSK9 (proprotein convertase subtilisin/kexin type 9) have emerged as a new class of drug to reduce LDL levels.[67] On the other hand, the levels of HDL-cholesterol are inversely correlated with the risk of cardiovascular disease.[68] Significant efforts have gone into the development of drugs that raise HDL-cholesterol levels, however such drugs have not shown a therapeutic benefit to date.[69] Nevertheless, both LDL and HDL enter atherosclerotic plaques and interact with cells there, rendering them of interest for plaque imaging or drug delivery.[70, 71] In addition, certain types of cancer overexpress the 
LDLr and take up LDL at a rate of up to fifty times higher than normal tissue.[72] Therefore LDL is an attractive platform to deliver drugs or imaging agents to these tumors. The high affinity HDL receptor SR-B1 is expressed in the liver, adrenals and in macrophages.[73] SR-B1 has been implicated in cancer and serves as a mechanism for HDL-based agents to target tumors.[74]

Currently, have been several HDL-based nanoparticles that have undergone clinical evaluation, most notably apoA-1 Milano, CER-001 and CSL-112 (recently reviewed in $[75,76])$. ApoA-1 Milano is a mutant version of apoA-1, with an amino acid substitution of arginine to cysteine at residue 173, whose expression correlates with decreased cardiovascular disease risk.[77] Recombinant apoA-1 Milano complexed with phospholipids, also referred to as ETC-216 or MDCO-216, has been tested in clinical trials. Administration of apoA-1 Milano/phospholipids weekly for 5 weeks significantly decreased the coronary atherosclerotic burden, as measured by intravascular ultrasound in a small clinical trial.[78] While these results are promising, especially given the relatively short length of the study, the ability to produce sufficient quantities of apoA-1 Milano has hindered its development as a therapeutic.[79] Two formulations of HDL, combining wild type apoA-1 with phospholipids, have progressed to clinical trials. CSL-112 (previously referred to as CSL-111) combines apoA-1 derived from human plasma with phosphatidylcholine isolated from soybeans to generate a reconstituted HDL mimetic (rHDL), with biological similarity to native HDL.[80] Intravenous administration of CSL-112 weekly for 4 weeks, similar to the ETC-216 study detailed above, did not alter atherosclerotic plaque burden compared to placebo, but did improve other measures, including the plaque characterization index and coronary score.[81] CSL-112 was well 
tolerated in healthy volunteers,[82] and induced enhanced cholesterol efflux, mediated through ABC-A1, after administration.[83] In a different clinical trial, CSL-112 was administered to type 2 diabetics, and shown to decrease platelet aggregation ex vivo, suggesting that CSL-112 may be an effective therapy for limiting vascular complications in individuals with type 2 diabetes.[84]

Similar to CSL-112, CER-001 is a reconstituted HDL mimetic, using recombinant apoA-1 rather than apoA-1 isolated from human plasma. In a trial similar to the apoA-1 Milano study but with a larger study population, CER-001 was given intravenously and atherosclerotic burden quantified. While CER-001 was well tolerated in the patients, no quantifiable benefit of CER-001 administration was observed compared to placebo control.[85] However, in a separate trial, CER-001 was found to decrease carotid artery wall thickness, which increases during the development of atherosclerosis, in patients with homozygous familial hypercholesterolemia,[86] implying that rHDL therapy may be more useful in patients where the current standard of care (e.g. LDL-lowering therapies) are insufficient to lower cardiovascular disease risk.

A major benefit of the injectable apoA-1 containing therapies is their potentially long circulating half-life. For these therapies, the circulating half-life of apoA-1 is on the order of 48-72 hours,[75] suggesting that after the initial burst of cholesterol efflux to rHDL and its subsequent conversion to cholesteryl esters for transport to the liver, the injected apoA-1 can be recycled into the endogenous pool of apoA-1 for further reverse cholesterol transport.

The ways in which lipoproteins can be modified for delivery of contrast media or therapeutics are shown in Figure 2. Individual examples will be discussed below, but we 
will give a broad overview of the available modifications here. First, most delivery platforms have been based on LDL or HDL since their circulation times in the blood and penetration into disease sites is greater than for the other lipoproteins. HDL is somewhat easier to modify than LDL, since HDL can be separated into its components and reconstituted with addition payloads, such as drugs or nanocrystals. This is because the apolipoproteins of HDL are composed of fewer amino acids than the major apolipoprotein of LDL (B-100), which irreversibly self-aggregates when isolated. Nevertheless, it has been shown that labeled lipids, drugs and even nanocrystals can be inserted into intact LDL.[39, 87, 88]

Lipoproteins are valuable as delivery platforms for a number of reasons. First, they have inherent biological importance, both in normal biological function and in diseases such as familial hypercholesterolemia and coronary artery disease. Second, the natural targets of lipoproteins (e.g. macrophages and the LDL receptor) are of considerable interest, both for imaging and therapeutic purposes. They are also highly biocompatible, biodegradable and non-immunogenic.[89] Next, it has been shown that lipoproteins can be re-routed to other targets via attachment of additional ligands to the apolipoprotein component[43] or the lipid coating.[90] Lipoproteins are also versatile, in that they can carry hydrophobic, lipophilic or hydrophilic payloads. This has allowed them to act as delivery agents for hydrophobic drugs,[44] amphiphiles labeled with gadolinium chelates to act as MRI contrast agents,[91] and nucleic acids attached to the lipoprotein surface.[92] Lipoprotein delivery platforms have been used in cardiovascular diseases, cancer, Alzheimer's, and others.[42, 93, 94] They have been used as imaging agents for MRI, CT, fluorescence imaging, PET and other methods.[42, 88, 95, 96] 
Highly lipoprotein-like nanoparticles can be made by using peptides in place of apolipoproteins.[97-99] Interestingly, it has been recently shown that PLGA, a biodegradable polymer frequently used in drug delivery systems, can be used as the core of HDL in place of triglycerides.[100] On a practical level, the materials needed to form lipoprotein delivery agents are easy to obtain, with the different lipoproteins available to purchase, as well as individual lipids and some of the apolipoproteins. Furthermore, lipoproteins can be isolated by sequential ultracentrifugation from human plasma (excess human plasma is frequently available from blood banks).[49] In addition, as alluded to above, peptides that mimic some of the apolipoproteins can be synthesized or purchased.[97, 101]

With the large number of modifications of lipoproteins available, and the plethora of studies published on nanoparticles, it is reasonable to ask the question, when is a nanoparticle a lipoprotein?[102] Given the characteristics listed for lipoproteins above, lipoprotein-based nanoparticles should have a coating largely composed of natural phospholipids, into which is embedded either apolipoproteins or peptide mimics of apolipoproteins. This coating should cover a hydrophobic core (optional for HDL). For example, an emulsion where a PEGylated phospholipid coated a triglyceride core, might have some general similarities to lipoproteins in terms of size, density and hydrophobic core. However, lack of an apolipoprotein or peptide apolipoprotein mimic and a predominately non-natural lipid coating indicate that this is not a lipoprotein-based nanoparticle. These were the criteria that we used to select studies to discuss in this review article. A criterion that could be used is that the nanoparticle should possess some functional aspect(s) of the natural lipoprotein it is mimicking, such as the ability to efflux 
or influx cholesterol or cholesteryl esters from cells, or bind to lipoprotein receptors. However, relatively few studies investigate such functionality, therefore we used a more expansive definition.

\section{Lipoprotein-based contrast agents}

\subsection{High density lipoprotein contrast agents}

\subsubsection{Labeled native HDL contrast agents}

HDL has been adapted as a contrast agent in numerous studies. The first report of HDL as an imaging agent was that of Shaish et al., who radiolabeled the protein components of HDL with I-125.[103] They used this radiolabeled HDL to study its clearance from blood in the apoE knockout (apoE KO) mouse model of atherosclerosis. They found the clearance to be relatively slow, with $30 \%$ of the dose still remaining in the blood at 24 hours (although it must be noted that this half-life is for the protein component which is recycled - others have shown the half-life of the lipids of HDL to be shorter, i.e. 2-3 hours).[44, 104] The authors also examined the biodistribution of HDL, finding that most of it was found in the heart, liver, kidney, aorta and lung at 24 hours. Last, autoradiography was done to visualize the accumulation of HDL in the aorta. HDL was shown to accumulate in the arch and in the abdominal aorta, close to the renal arteries and bifurcation, areas where significant amounts of plaque typically develop.

\subsubsection{Reconstituted HDL contrast agents}

Subsequently, a group led by Fayad, Mulder and Fisher reported a large number of studies where HDL was adapted as a contrast agent for imaging techniques such as 
MRI, CT, fluorescence and PET.[40, 91, 94-96, 105, 106] The approach for these agents was to disassemble HDL into its protein and lipid components and then reconstitute it with a variety of payloads that provide image contrast. Interestingly, this group recently reported a novel method of HDL reconstitution where a microfluidic chip was used to form HDL in a fast, one step procedure, which may facilitate scale up of HDL synthesis.[107] This group's first papers on this topic focused on including gadolinium labeled and fluorescent lipids in the nanoparticle in order to create a contrast agent active for MRI and fluorescence imaging methods.[91, 94] This agent was injected into apoE KO mice. MRI scans performed post-injection revealed significant increases in contrast in the aortas of these mice. Confocal microscopy and histology showed that this labeled HDL accumulated in macrophage-rich areas of the plaque, which is of interest since macrophages have been linked to plaque instability and risk of heart attacks.[63] A number of steps were taken to enhance this platform, such as the use of higher relaxivity gadolinium chelates to label the lipids.[108] In addition, it was reported that the inclusion of a lipopeptide that was based on the LDLr binding domain of apoE resulted in greater uptake in macrophages in vitro than without the lipopeptide and also resulted in stronger MRI contrast in vivo.[109] Other groups have confirmed the utility of HDL labeled with gadolinium chelating lipids as MR contrast agents.[110, 111] Additional approaches to form gadolinium-labeled HDL include attachment of gadolinium chelates to apoA-I and inclusion of gadolinium chelates conjugated to cholesterol in the lipid layer.[112-114] Recently, Wang et al. reported a europium labeled HDL nanoparticle (using a phospholipid that was conjugated to a europium chelate) that produced contrast for a type of MRI known as chemical exchange saturation transfer (CEST).[115] 
In 2009, the Zheng lab reported reconstituted HDL loaded with bacteriochlorin $e 6$ bisoleate, a fluorophore that emits in the near-infrared (NIR) region of the visible spectrum.[116] Tissue transmits light relatively well in the NIR region (650-900 nm) and therefore fluorescence imaging systems typically operate in these wavelengths.[117] The authors showed that these nanoparticles were taken up in the KB cancer cell line that expressed the SR-BI receptor (a receptor for HDL).[60] It was also shown that the nanoparticles accumulated in tumors that formed when KB cells were inoculated in the flank of mice, via fluorescence imaging.

Most recently, HDL has been developed into an agent for PET imaging. A study from the Reiner group used deferoxamine conjugated either to phospholipids or apoA-I to complex the radioisotope $\mathrm{Zr}-89$ for PET contrast.[95] The fluorophore DiO was included to allow detection by fluorescence methods. The agent was administered to mice bearing breast tumors and was found to avidly accumulate in the tumors at up to $17 \%$ injected dose/gram tissue. Microscopy and flow cytometry showed that the nanoparticles preferentially bound to tumor-associated macrophages, an important cell type in cancer.

\subsubsection{Peptide mimetic HDL contrast agents}

Interest in HDL-based therapeutics has stimulated many years of research into synthetic peptides that can mimic the properties of apoA-I. A number of peptides (e.g. $18 \mathrm{~A}, 37 \mathrm{pA}, \mathrm{D} 4 \mathrm{~F})$ have been developed that form amphiphatic $\alpha$-helices and interact with lipids to form HDL-like or HDL-mimicking nanoparticles.[97, 118] These nanoparticles recapitulate many of the features of HDL, such as size, shape, macrophage cholesterol efflux and binding to ABC-A1. Cormode et al. reported HDL-like nanoparticles formed 
from natural phospholipids, gadolinium labeled phospholipids, fluorescent lipids and either $18 \mathrm{~A}$ or $37 \mathrm{pA} .[98,104]$ The authors found that these nanoparticles were taken up by macrophages in vitro in a saturable, receptor-like manner, similar to labeled nanoparticles formed from native HDL. In vivo imaging experiments with MRI also showed that these nanoparticles accumulated in the macrophages in the aortas of apoE KO (atherosclerotic) mice, indicating that synthetic HDL nanoparticles performed comparably with native HDL.

The Zheng lab reported the formation of HDL-like nanoparticles loaded with the NIR fluorophore known as DiR (1,1'-Dioctadecyl-3,3,3',3'tetramethylindotricarbocyanine iodide).[99] These nanoparticles were formed using the apoA-I mimicking, amphiphatic peptide 18A. The authors found that these nanoparticles were taken up by cells that express the SR-BI receptor in vitro and in tumor models by fluorescent imaging, as well as demonstrating direct cytostolic delivery of the payload. DiR-labeled HDL has also been used to image the macrophage content in atherosclerotic lesions.[119] In another study from the Zheng group, HDL-like nanoparticles were formed using natural phospholipids, porphyrin-labeled phospholipids and R4F, an apoA-I mimicking peptide, creating a nanoparticle $20 \mathrm{~nm}$ in size (Figure 3A, B).[120] The radioisotope $\mathrm{Cu}-64$ can be complexed in the porphyrin groups, to make the nanoparticle PET-active. The porphyrin group also renders the nanoparticle active for PDT and NIR fluorescence imaging. PDT is a cancer treatment modality where molecules known as photosensitizers that have accumulated in the tumor are irradiated with light. The light interacts with the photosensitizer to generate singlet oxygen, which kills the nearby tumor cells. Injection of the agent in mice bearing glioma tumors, combined with laser 
irradiation, resulted in significant cell death in the tumors via PDT, as assessed by a form of histology known as TUNEL (terminal deoxynucleotidyl transferase dUTP nick end labeling) staining (Figure 3C). This agent was shown to permit the detection of prostate cancer via both PET/CT and fluorescence molecular tomography imaging (Figure 3D,E). Furthermore, the agent was used to aid in tumor resection via intra-operative imaging, as shown in Figure 3F.

\subsubsection{Nanocrystal labeled HDL contrast agents}

Another development in the field of HDL contrast agents was the inclusion of nanocrystals in the core of HDL.[40] This was achieved by use of various hydrophobically-coated nanocrystals such as gold, iron oxide or quantum dots. The nanocrystals were co-dissolved with lipids in chloroform and dropped into hot water, forming a suspension of the nanocrystals in lipid micelles. Addition of apoA-I to the resulting solution, followed by purification, yielded HDL-like nanoparticles with single nanocrystals encapsulated in the core. Inclusion of fluorescent (rhodamine) and/or gadolinium labeled lipids rendered these nanoparticles active for several modalities. Multimodal contrast agent nanoparticle formulations have the potential for enhanced imaging through synergies achieved by combining data from multiple imaging techniques [recently reviewed in [121]]. For example, HDL with a gold core and fluorescent and gadolinium labeled lipids in the coating were shown to provide contrast for CT, MRI and fluorescence imaging techniques (contrast in CT is provided by elements that have high atomic numbers, such as gold, iodine, bismuth, etc). Nanocrystal loaded HDL targeted macrophages in atherosclerotic plaques similarly to the formulations described above. 
Iron oxide core HDL has been probed in detail for its biological interactions.[105] The quantum dot core HDL was modified with a Cy5.5 lipid fluorophore such that Förster resonance energy transfer (FRET) occurred between the quantum dot and the Cy5.5.[106] This system was used to study the biointeractions of the HDL nanoparticle with other lipoproteins and cells. Last, the gold core HDL was used as a contrast agent for a novel CT imaging technique known as spectral CT.[96] In this type of CT, the contrast arising from different materials can be distinguished. It was shown that gold, iodine, calcium and soft tissue could be distinguished, which could be highly valuable for determining atherosclerotic plaque composition.

\subsubsection{Re-routed HDL contrast agents}

In all the studies mentioned above, HDL-based platforms were used to deliver contrast media to the natural targets of HDL in either cardiovascular disease or cancers. However, it has also been shown that HDL can be redirected from its natural targets to alternative targets.[42, 90, 122] Chen et al. conjugated the arginine-glycine-aspartic acid (RGD) peptide to apoA-I on HDL labeled with gadolinium and the NIR fluorophore DiR. In vitro experiments with activated endothelial cells and macrophages, showed that the nanoparticles had been successfully re-routed to the $\alpha_{v} \beta_{3}$-integrin (the target for RGD), since the nanoparticles bound preferentially to the endothelial cells, which overexpress the $\alpha_{v} \beta_{3}$-integrin. MRI and fluorescence imaging studies revealed that the re-routed HDL more rapidly accumulated in tumors (where $\alpha_{\mathrm{v}} \beta_{3}$-integrin expression is high) than control HDL. Confocal microscopy showed that the RGD-targeted HDL localized at endothelial cells in the tumors at 1 hour post-injection, proving that the targeting effect occurred in 
vivo. A similar platform was re-routed to collagen and used to image the collagen content in atherosclerosis with MRI (in atherosclerosis collagen is a marker for plaque stability).[90] Endothelial growth factor (EGF) conjugation to fluorescent HDL nanoparticles was used to distinguish tumors that overexpress the EGF receptor from those that do not.[123] Folate has been used to re-route HDL to ovarian cancer (where the folate receptor is overexpressed), as demonstrated by fluorescence imaging.[122] Interestingly, the authors found that the most successful route of administration was via intraperitoneal injection rather than intravenous injection. Another study, using iron oxide core HDL, also showed that intraperitoneal injection was viable to perform imaging with HDL-based agents.[124] This indicates that use of these agents may be streamlined by use of via intraperitoneal injections,[89] which is more straightforward than intravenous injection.

\subsection{Low density lipoprotein contrast agents}

\subsubsection{Labeled native LDL contrast agents}

Adaption of LDL as a contrast agent is also an active area of research. Similar to HDL, the first work in this area involved radiolabeling LDL and performing imaging with nuclear techniques.[103, 125-128] A number of different radionuclei have been used to labled LDL, i.e. I-123, I-125, In-111, F-19 and Tc-99m. For example, Iuliano et al. labeled oxidized LDL (oxLDL) with Tc-99m.[129] They found that the Tc-99m-oxLDL was cleared from the blood more rapidly than native LDL and that uptake in the liver was more extensive. Furthermore, gamma camera images of patients with carotid artery 
disease revealed significantly more uptake of Tc-99m-oxLDL in the plaque compared to controls.

LDL was subsequently adapted for use as a fluorescence imaging agent as reported in a series of publications in the early 2000s.[130-132] For example, native LDL was labeled with a lipophilic fluorophore named DiI by incubation (DiI-LDL).[132] Binding of DiI-LDL to LDLr was proven via fluorescence microscopy experiments with differing concentrations of DiI-LDL and with an excess of unlabeled LDL. Fluorescence imaging was performing using B16 and HepG2 tumor models (both types of cancer cells that overexpress the LDLr). Uptake was observed in both tumor types, although accumulation was found primarily at the tumor rim in B16 tumors, whereas the distribution was more uniform in HepG2 tumors.

LDL has also been adapted to act as an MRI contrast agent. The first study in this area used managanese ions complexed in a hydrophobic porphyrin molecule to label LDL via incubation.[133] Manganese has five unpaired electrons and is therefore quite paramagnetic and has often been considered as an element upon which to base MRI contrast agents.[134] This Mn-LDL was incubated with foam cells (a cell type that avidly takes up LDL in atherosclerotic plaques). MR imaging of pellets of these cells revealed that the cells incubated with Mn-LDL gave a stronger signal than cells incubated with unlabeled LDL, indicating the efficacy of Mn-LDL as an MRI contrast agent. LDL has also been labeled with gadolinium chelates. Li et al. labeled LDL with a gadolinium chelate conjugated to a fluorophore (similar in structure to Cy3.5), via incubation.[135] The authors demonstrated the efficacy of the agent via in vitro fluorescence microscopy and in vivo MRI experiments. $T_{1}$ MR maps of B16 tumors (a cell type that overexpresses 
the LDLr) indicated the uptake of the Gd-LDL in the tumors at 16 hours post-injection. The same group also labeled LDL using gadolinium chelate conjugated lipids.[136] More recently this group has applied this platform for imaging plaque in a mouse model of atherosclerosis $[137,138]$ The Aime group labeled LDL with lipids whose headgroup contained either rhodamine or a gadolinium chelate known as AAZTA.[88] This chelate gives almost twice the MRI contrast of a typical gadolinium chelate since two water molecules can be coordinated instead of one.

\subsubsection{Re-routed LDL contrast agents}

The next major advance in the field of LDL contrast agents was the development of LDL re-routed to targets other than the LDLr. In 2005, Zheng et al. reported LDL that was fluorescently labeled with DiI and modified with folate attached to the lysine residues of LDL.[43] The authors showed via in vitro studies that the nanoparticle had been successfully re-routed to the folate receptor. In a follow-up study, the same group reported folate re-routed LDL that was loaded with the fluorophore DiR (a fluorophore similar in structure to DiI, but with emission in the NIR).[139] The authors demonstrated the targeting of the folate-conjugated LDL in an elegant in vivo imaging experiment. Two types of tumors were inoculated in mice, one that expressed the folate receptor and another type that does not. Greater contrast was observed in the tumor that overexpressed the folate receptor than in the tumor that did not, confirming the targeting effect in vivo.

\subsubsection{Reconstituted LDL contrast agents}


Reconstitution of LDL is a much less favored approach than for HDL, since it is a more difficult process. Nevertheless, reconstituted LDL contrast agents have been reported. For example, Hill et al. synthesized iodinated triglycerides to label LDL to form a contrast agent for $\mathrm{x}$-ray based imaging techniques such as CT.[140] Labeling was done via the Krieger method,[141] which involves lyophilization of LDL, extraction of the original triglyceride core, addition of the iodinated triglycerides in toluene, removal of the solvents and reconstitution of the LDL in buffer. CT imaging of solutions of this labeled LDL and of pellets of HepG2 cells that had been incubated with the labeled LDL demonstrated that this formulation could act as a CT contrast agent.

\subsubsection{Nanocrystal labeled LDL contrast agents}

Allijn et al. recently reported a method to load LDL with nanocrystals of various types (i.e. gold, iron oxide or quantum dots) or other hydrophobic substances, such as fluorophores (i.e. BODIPY [boron-dipyrromethene] and DiR).[39] This was done by first suspending the nanocrystal or fluorophore in a micelle formed from the phospholipid myristoyl-hydroxy-phosphatidylcholine. These micelles were sonicated together with native LDL, which resulted in transfer of the nanocrystal or fluorophore cargo into the core of LDL (Figure 4A). Via inclusion of both nanocrystals and fluorescent lipids in the micelles, LDL active for multiple imaging modalities could be formed, such as gold and rhodamine labeled LDL, which was active for both $\mathrm{CT}$ and fluorescence techniques. The authors focused on gold core LDL (Au-LDL) for most of the experiments. Negative stain electron microscopy confirmed the loading of gold cores into LDL (Figure 4B-D). In vitro fluorescence microscopy experiments showed that B16-F10 cells (a melanoma cell 
line that overexpresses the LDL receptor) avidly took up Au-LDL, but that the uptake was reduced when with incubated with Au-LDL and an excess of unlabeled LDL (Figure 4E-G). This experiment showed that Au-LDL was specifically taken up by the LDL receptor. Furthermore, in vivo experiments showed that Au-LDL circulated approximately twice as long in LDL receptor knockout (LDLr KO) mice than wild type mice (Figure 4H). This confirmed that Au-LDL was functioning in a similar fashion to native LDL, since a lack of the LDLr would reduce the rate of clearance of LDL from the blood as has been previously reported.[142] In vivo tumor imaging was performed with this agent using CT, fluorescence imaging and spectral CT. As can be seen in the spectral CT images, Au-LDL accumulation in the tumor of a mouse (Figure 4I) and in the liver (Figure 4J), an important organ for LDL clearance, could be observed. Ex vivo TEM on tumor tissue confirmed the uptake of Au-LDL.

\subsection{Very low density lipoprotein contrast agents}

VLDL has only rarely been adapted for use as a contrast agent. It has been labeled with I-123 for nuclear imaging techniques.[143] Such radiolabeled VLDL was studied in normal and Watanabe-heritable hyperlipidemic (WHHL) rabbits, a strain of rabbit that does not have the LDLr. Radiolabeled VLDL was taken up in the liver of WHHL rabbits more slowly than in normal rabbits, indicating the importance of the LDLr for hepatic uptake of VLDL in rabbits. More recently Barazza et al. labeled VLDL with fluorescent or gadolinium-chelate lipids, to form a contrast agent active for MRI and fluorescence.[144] In this study, the authors were investigating the in vivo mechanism of contrast generated from gadolinium labeled lipoproteins. It was found that there was 
significant transfer of the gadolinium label to LDL and VLDL from HDL, indicating a role of LDL and VLDL in the delivery to target tissues.

\subsection{Chylomicron contrast agents}

Chylomicrons have also only rarely been studied as contrast agents. While they have been radiolabeled and labeled with gold nanoparticles, no imaging has been done with such agents, to the best of our knowledge.[145, 146] However, in 2009, Bruns et al. published a study where chylomicron-based nanoparticles were formed that were labeled with quantum dots and iron oxides in the core, creating contrast for fluorescence and iron oxides, respectively.[147] Chylomicron-like emulsions loaded with these diagnostically active nanocrystals were formed from lipids and triglycerides extracted from native lipoproteins. Incubation with ApoE and lipoprotein lipase gave the nanoparticles chylomicron-like properties. This platform facilitated the study of the metabolism of chylomicrons via fluorescence imaging and MRI.

\section{Drug delivery with lipoproteins}

\subsection{Drug delivery with high density lipoprotein}

There is considerable interest in utilizing HDL as a drug delivery vehicle, particularly for new anti-cancer drugs. A large percentage of anti-cancer therapeutics that have been developed do not specifically target malignant cells; rather, the drugs indiscriminately affect both malignant and healthy tissues, leading to harsh side-effects and poor cure rates. Due to their size, nanoparticles frequently preferentially accumulate in tumors due to leaky vasculature, a phenomenon known as the enhanced penetration 
and retention (EPR) effect,[148-150] although, solid tumors are heterogeneous, and the EPR effect does not always occur.[151] Furthermore, increasing nanoparticle circulation time increases tumor accumulation.[152] Since HDL and LDL are in the right size range for extravasation in tumors ( $400 \mathrm{~nm}$ or less, depends on the tumor type) and have lengthy circulation times, they are well suited to deliver substantial drug payloads to tumors via the EPR effect. Beyond any potential EPR effect, HDL may be actively targeted to malignant cells, depending on the tumor type. Due to their reliance on cholesterol and cholesteryl esters, the HDL receptor SR-B1 is upregulated in numerous malignancies,[153-158] providing an attractive target for drug delivery. Recent reports have demonstrated the ability of both discoidal HDL, and mature, spherical HDL to bind to SR-B1.[159] Uptake of HDL cargo, including anticancer drugs, occurs through a nonendocytic pathway.[74, 160] This is highly desirable for drug delivery, as endosomal uptake of drug-loaded nanoparticles typically leads to lysosomal degradation of both the drug and the delivery vehicle.

As mentioned above, lipoproteins possess a hydrophobic core, which can be loaded with drugs that have poor water solubility. It has been estimated that $\sim 40 \%$ of new therapeutics have solubility issues,[161] limiting the amount of drug that can be administered. Incorporation of drugs with solubility issues into the hydrophobic core of HDL allows for an increase in the concentration of drug that can be administered.

In a recent report, the anti-cancer drug paclitaxel, a widely used chemotherapeutic, was loaded into discoidal HDL synthesized with monocholesteryl succinate (CHS).[162, 163] Discoidal HDL interacts with cholesteryl ester transfer protein (CETP), leading to increased cholesteryl ester content and maturation into 
spherical HDL. This transformation can lead to release of some of the drug cargo;[164] therefore, CHS was incorporated into the synthesis to maintain the discoidal conformation of the HDL after contact with serum. The modified discoidal HDL increased the circulation half-life of paclitaxel in rats, and showed increased efficacy in breast cancer tumor xenografts compared to liposomal or free paclitaxel.[162, 163]

Spherical HDL has been used as a drug delivery vehicle, typically by loading the lipophilic core with drugs with poor water solubility. In a recent report, Lacko et al. sequestered the poorly water soluble drug valrubicin into spherical HDL.[74, 165] This drug loaded HDL had significantly increased toxicity against SR-B1 expressing ovarian and prostate cancer cell lines, compared to valrubicin alone. Drug toxicity in normal prostate and ovarian epithelial cell lines was reduced in the HDL-drug formulation compared to drug alone. Similar work has been reported, with HDL loaded with fenretinide,[165] a retinoid derivative, and doxorubicin.[166, 167] In both cases, encapsulation of the drug in HDL enhanced cytotoxicity of neuroblastoma and hepatocellular carcinoma cells, respectively, while reducing off-target effects in healthy, normal cells.

Asides from anti-cancer drugs, HDL has been used to encapsulate other lipophilic drugs, such as rapamycin.[168] Rapamycin, also known as sirolimus, has seen extensive use in organ transplantation as an immunosuppressive drug, as well as in coronary stent coating to prevent neointimal hyperplasia following vascular intervention. Rapamycin is poorly soluble in water, limiting the effective dose that can be delivered. Incorporation of rapamycin into a recombinant HDL decreased cellular senescence in primary human dermal fibroblasts and human umbilical vein endothelial cells in vitro, and demonstrated 
a stronger anti-inflammatory activity in vivo, compared to rapamycin alone or reconstituted HDL alone.[169]

More recently, Duivenvoorden et al. reported simvastatin loaded HDL ([S]rHDL), which was used as a treatment for atherosclerosis.[44] While statins are widely used to lower LDL-cholesterol levels, when taken as an oral drug their effects are primarily in the liver. Formulation into an injectable HDL nanoparticle allows delivery of the statin to atherosclerotic plaque for direct local action. Uptake in plaque was evidenced by additionally loading the HDL nanoparticle with gadolinium chelates and DiR/Cy5.5 fluorophores and performing MRI or fluorescence imaging, as can be seen in Figure 5B,C. The therapeutic effect of this treatment was assessed via MR imaging of plaque volume, histology and mRNA analysis. Sample histology images and quantification of thousands of such images are displayed in Figure 5D-F. As can be seen, from a single week of high dose [S]-rHDL infusions, the plaque area was significantly reduced and CD68 staining (a macrophage marker) was very substantially reduced. In a follow-up study, the mechanism of action was found to be inhibition of macrophage proliferation, rather than inhibition of monocyte recruitment, as well as suppression of inflammation in resident macrophages.[170] Furthermore, it was demonstrated that the combination of a brief period of [S]-HDL injections followed by oral statin therapy was an effective therapeutic approach to control atherosclerosis.

While SR-B1 is the major receptor for HDL, incorporation of various targeting moieties allows for HDL uptake through non-SR-B1 receptors. As mentioned above, HDL has been modified by covalently adding folate and other targeting ligands to the lysine residues in apoA-I.[42, 90, 122, 171] One can imagine that similar preferential 
targeting to folate receptor-alpha positive malignant cells would be seen in anti-cancer drug loaded folate-containing HDLs.

\subsection{Drug delivery with low density lipoprotein}

Low density lipoprotein is attractive as a drug delivery vehicle for both cancer and atherosclerosis due to the prevalence of the LDLr on malignant cells and the macrophages that comprise atherosclerotic plaques. As with HDL, drug payloads can be encapsulated in the hydrophobic core of LDL or incorporated into the amphipathic phospholipid layer.[172]

Dexamethasone has been investigated as a therapeutic to prevent atherosclerotic plaque development, $[173,174]$ however, prolonged dexamethasone administration is associated with significant adverse side effects.[175] Tauchi et al. incorporated dexamethasone into LDL, improving delivery to atherosclerotic plaques and reducing progression of atherosclerosis in a mouse model.[176, 177] Loading this drug in LDL ameliorated the adverse side effects observed from repeated systemic administration of dexamethasone.

Drug loading of LDL for cancer has involved common, broad spectrum chemotherapeutics, such as doxorubicin[178] and paclitaxel,[179] as well as 5fluorouracil (5-FU).[180] In each of these cases, drug loading into or onto LDL targeted the therapeutic to cancer cells and improved efficacy compared to free drug while also decreasing off target toxicity.

Finally, similar to HDL, targeting moieties can be conjugated to LDL to shift the targeting of the nanoparticles from the LDLr to a different receptor. Meng et al. reported 
on the use of lactoferrin to target curcumin loaded LDL (Lf-mNCL) to deliver therapeutics to brain cells to delay the progression of Alzheimer's disease.[181] The LfmNCL was able to successfully cross the blood brain barrier and mitigate the damage associated with Alzheimer's disease in a rat model. The ability of drug loaded LDL to cross the blood brain barrier offers the opportunity to deliver therapeutics to an immune privileged site while still retaining the benefits of drug encapsulation in lipoproteins.

\subsection{Drug delivery with very low density lipoprotein}

VLDL has rarely been studied for drug delivery purposes. In circulation, VLDL have relatively short half-lives (3-5 hours)[182], and interact with a host of proteins that alter the composition of both the hydrophobic core and amphipathic coating.[54] Kader and Pater compared the ability of VLDL, LDL, and HDL to deliver hydrophobic anticancer drugs, including 5-FU, doxorubicin, vincristine and 5-iododeoxyuridine.[183] They found that formulations with HDL and LDL enhanced the toxicity of the chemotherapeutics against the breast cancer cell line MCF7 compared with free drug controls, while incorporation into VLDLs did not alter drug toxicity. The authors attributed this to inefficient internalization of VLDLs by the breast cancer cells. Given the prevalence of the LDLr and SR-B1, the high affinity receptors for LDL and HDL, respectively, on cancer cells, VLDL appears to be a poor fit for drug delivery.

\subsection{Drug delivery with chylomicrons}

Chylomicrons have short circulating half-lives,[184, 185] making them ill-suited as stand-alone drug delivery vehicles. Rather, recent lines of investigation have utilized 
chylomicrons as a secondary delivery method to prolong bioavailabilty and circulating half-lives for nanoparticle-encapsulated drugs after oral ingestion.[186, 187] In a recent study, Makwana et al. demonstrated that a significant percentage of orally administered Efavirenz, an antiretroviral drug, encapsulated in solid lipid nanoparticles was able to escape the first pass metabolism by the liver.[188] The drug was found in the lymphatic system, having been internalized in chylomicrons. The solid lipid nanoparticles not only evaded metabolism by the liver but were targeted, via the chylomicron, to the lymphatics,[188] which act as a viral reservoir in HIV-infected patients and is typically inaccessible to therapeutic interventions.[189]

\section{Nucleic acid delivery with lipoproteins}

RNA interference (RNAi) has incredible therapeutic potential across a wide range of diseases, and allows for the targeted knockdown of a specific gene. Several different RNAs, from short, interfering RNA (siRNA), short hairpin RNA (shRNA) and microRNAs (miRNA), can be utilized in RNAi.[190] While the ability to tailor RNAi therapeutics to specifically target a single gene is powerful, the resultant RNA molecules are inherently unstable, leading to the need for a mechanism to deliver the therapeutic RNA to the cytoplasm of target cells.[190-192] A variety of modifications have been made to the structure of RNA (reviewed in [193]) in order to improve nucleic acid stability and bioavailability in vivo. Conjugation of a cholesterol molecule to the 5' or 3' end of the antisense strand of siRNA allows the nucleic acid to bind to albumin and lipoproteins, such as HDL and LDL, prolonging the circulating half-life and improving the stability of the siRNA.[194-197] In vivo, systemically administered cholesteryl- 
conjugated siRNAs are targeted preferentially to the liver[196, 198, 199], due to their binding to serum lipoproteins such as HDL, and trafficking to cells expressing SRB1.[197]

Vickers, et al. have recently demonstrated that natural HDLs, isolated from human serum, contained miRNAs, and that these HDL-bound miRNAs displayed improved stability over naked miRNAs.[41] HDL appears to be the preferred lipoprotein carrier for miRNAs, as the miRNA content of LDLs was significantly lower, in both healthy patients and those with cardiovascular disease.[200] HDL's role as a natural nucleic acid carrier makes it a highly attractive nanostructure for the delivery of therapeutic nucleic acids in vivo. There have been two major approaches to HDL mediated siRNA delivery, namely the use of either reconstituted HDL or synthetic HDL mimetics.

Reconstituted HDLs (rHDL), composed of apoA1, phospholipids, cholesterol and cholesteryl esters, have shown promise in siRNA delivery. Incorporation of siRNA into reconstituted HDLs by Shahzad et al. resulted in nanoparticles with a similar size to natural HDL, and that are effective for knocking down target gene expression.[158] The rHDL-siRNA nanoparticles shown to be targeted to SR-B1, and were preferentially taken up by SR-B1 expressing tissues compared to SR-B1 negative tissues in vivo. Further, as mentioned above, SR-B1 is over-expressed in a variety of malignancies, as well as in numerous human cancer cell lines.[153-158, 201] In vivo, systemic administration of rHDL-siRNA nanoparticles resulted in effective target gene regulation in both colorectal cancer and ovarian cancer models.[202]

An approach combining nucleic acid delivery facilitated by cationic lipids and 
recombinant HDL was recently reported.[203] The authors refer to the particles as nanodisks (ND) due to the addition of bioactive reagents, namely the cationic lipid and siRNA, which are not typical of recombinant, discoidal HDL. The data demonstrated that NDs formulated with 1,2-dimyristoyl-sn-glycero-3-phosphocholine (DMPC), the cationic lipid, 1,2- dimyristoyl-3-trimethylammonium-propane (DMTAP), and apoA-I were able to bind and stabilize duplex siRNA. In proof-of-concept experiments, NDs carrying siRNA targeting GAPDH (glyceraldehyde 3-phosphate dehydrogenase) were shown to reduce target gene expression in hepatoma cells by $60 \%$.

To generate a synthetic HDL mimetic, two different scaffold materials, a gold nanoparticle and HDL-mimicking peptide-phospholipid scaffold, have been utilized. First, bio-inspired HDL nanoparticles have been made using $5 \mathrm{~nm}$ diameter gold nanoparticles surface functionalized with apoA-I and phospholipids (Figure 6A).[204] These HDL nanoparticles have a similar shape, size, and composition as native mature, spherical HDLs, with $\sim 3$ copies of apoA-I per nanoparticle and an outer phospholipid layer consisting of 1,2-dipalmitoyl-sn-glyerco-3-phosphocholine (DPPC).[204, 205] Functionally, these HDL nanoparticles have been shown to engage SR-B1, as well as $\mathrm{ABC}-\mathrm{A} 1$ and $\mathrm{ABC}-\mathrm{G} 1$, and mediate cholesterol flux through the receptors in macrophages in vitro.[204, 205] These HDL nanoparticles were able to adsorb cholesterylated antisense DNA (chol-DNA) onto their surface, and deliver the functional nucleic acid to SR-B1 expressing cells in vitro to modify gene expression.[92] No adverse effects on cell viability were found from these nanoparticles (Figure 6B). With a nucleic acid cargo directed against miR-210, the levels of this microRNA were reduced by $80 \%$, comparing favorably with the $55 \%$ knockdown produced by the free nucleic acid 
(Figure 6C). Furthermore, this treatment resulted in upregulation of E2F3a, a target of miR-210 (Figure 6D). Cellular internalization of these chol-DNA HDL nanoparticles revealed that they were taken up in an endolysosomal-independent manner, avoiding a major hurdle in cellular nucleic acid delivery. These HDL nanoparticles were also shown to bind cholesterylated RNA, which was effective at reducing the cellular expression of vascular endothelial growth factor receptor 2 (VEGFR2).[206] Systemic administration of these conjugates resulted in decreased VEFGR2 expression in SR-B1 expressing cells, leading to decreased angiogenesis and a marked reduction in the volume of lung tumor xenografts.[206] Regardless of the type of nucleic acid adsorbed to the surface of this HDL platform, no toxicity was observed in normal, healthy cells and tissues both in vitro and in vivo. $[92,206]$ These studies indicate that gold core HDL is an excellent carrier for therapeutic nucleic acids.

In addition of a gold nanoparticle core, HDL mimetics can be synthesized using a peptide-phospholipid scaffold (HPPS).[207] Amphipathic peptides with $\alpha$-helical structures similar to apoA-I are combined with phospholipids and cholesteryl oleate to form self-assembled nanoparticles similar to serum-derived HDL.[207] HPPS were loaded with chol-siRNA targeted against bcl-2, a prominent oncogene in a wide array of cancers. Chol-siRNA HPPS were able to successfully deliver functional siRNA to the cytoplasm of SR-B1 expressing KB cells, a HeLa cell variant, but not to HT1080 cells, which only minimally express SR-B1.[207] As with the previously mentioned gold core HDL nanoparticles,[92] HPPS delivered nucleic acids independent of the endolysosomal pathway. 
While HDL has been the predominant lipoprotein investigated in nucleic acid delivery, some reports have emerged in which LDL was used as the delivery vehicle.[208, 209] LDLs pre-loaded with cholesteryl-siRNA were able to deliver these nucleic acids to LDLR expressing cells; however, it appears that the siRNA-LDL complexes are internalized via the endolysosomal pathway, providing for poor delivery of siRNA to the cytoplasm of cells.[208] Complexation of siRNA loaded LDLs with drug loaded N-succinylchitosan nanoparticles resulted in delivery of functional siRNA to liver tumor cells in vivo;[209] however, this nanoparticle formulation was internalized through the endolysosomal pathway, again potentially limiting the amount of functional, available siRNA that can be delivered. Finally, Kuwakara reported that cholesteryl-siRNA loaded LDLs did not improve deliver of siRNA to brain capillary endothelial cells in vivo compared with free siRNA, while siRNA loading onto HDL did result in improved delivery, including delivery to cells beyond the blood brain barrier.[210] These reports suggest that, while LDL has shown efficacy as a small molecule drug delivery vehicle, its delivery efficacy is limited in the case of therapeutic nucleic acids.

\section{Boron-neutron capture therapy with LDL}

An Italian group has published a number of studies based around cancer treatment via boron-neutron capture therapy (BNCT).[211-214] This is an experimental cancer therapy approach, where a non-radioactive isotope of boron (B-10) is delivered to the tumor.[215] This isotope captures neutrons at a rate many times higher than that of endogenous nuclei. The capture of a neutron results in fission of boron and damage to the tumor tissue. Geninatti-Crich et al. reported LDL that had been labeled with a lipid that 
has a gadolinium chelate in the head group, which is connected to a carborane (ten boron atoms) and a hydrocarbon chain.[213] Labeling was mediated with cyclodextrin, which served to disperse the gadolinium/carborane lipid micelles and make the lipid available to insert into LDL. This platform was studied in B16 tumor bearing mice. The gadolinium provided MRI contrast demonstrating the uptake of the labeled LDL in the tumor. Neutron irradiation of these mice resulted in highly reduced tumor growth compared with controls. Therefore this is a theranostic agent, since it combines both therapeutic and diagnostic properties. Subsequent developments include improved syntheses of the carborane lipids $[211,214]$ and success in use of carborane labeled LDL in conjunction with neutron irradiation to treat lung cancer.[212]

\section{Discussion}

In the past decade there has been tremendous progress and innovation in the field of lipoprotein delivery vehicles. The array of applications for lipoproteins is now very broad, with nucleic acid delivery, BNCT, CT and PET added in the past ten years.[40, 92, $95,213]$ The range of diseases that lipoproteins have been applied to has widened to include atherosclerosis, cancer and Alzheimer's.[42, 93, 94] The targets of lipoproteins have increased in number from their natural targets, to include the folate receptor, the $\alpha_{v} \beta_{3}$-integrin and collagen.[42, 90, 139] The expansions in applications and targets have been facilitated by innovations in the synthesis of labeled lipoproteins, such as methods to insert nanocrystals in the core of lipoproteins [39, 40, 147] or microfluidic synthesis of HDL.[107] For example, the development of insertion of gold nanoparticles into lipoproteins has led to in vivo applications in CT.[39, 40, 96] A number of studies have 
reported theranostic lipoproteins, where both therapeutic and diagnostic properties are included in the same agent. These include agents active for fluorescence imaging and PDT,[45] agents that provide contrast for MRI and regress atherosclerosis via localized statin delivery,[44, 170] or provide contrast for MRI and treat tumors via BNCT.[212]

Since there are numerous groups active in the area of lipoprotein delivery vehicles and due to the enduring importance of these natural nanoparticles, we expect that the next ten years will see much additional progress. Can lipoproteins be adapted to function for other applications such as photoacoustic imaging, SERS imaging, ultrasound, radiosensitization, in vitro diagnostics or as biomaterials? Both radiosensitization and SERS imaging could be achieved using modifications of gold core HDL, for example. Photoacoustics-active lipoprotein agents could be formed by inclusion of molecules that absorb in the NIR region. Will we see use of lipoprotein vehicles in additional diseases and directed against additional targets? Given the utility of lipoproteins in imaging and drug delivery to date, we expect expansion of the diseases and targets lipoproteins are directed against. Clinical trials of some of the agents developed in the past ten years would be expected. Underpinning these advances will likely be new particle components such as new peptide mimics of apolipoproteins and new materials with which to label lipoproteins. Novel methods to form labeled lipoproteins will probably emerge, and may include streamlined approaches such as in vivo labeling via injectable or orally consumable materials.

While there are many potential avenues for progress in this area, challenges remain in the field. Among the panoply of formulations that have been reported, which hold the most promise for clinical translation? Once agents have been selected, what 
method would be best to synthesize them? Lipoproteins could be isolated from individual patients, labeled and then re-administered to the same patient, however this approach would likely be cumbersome and expensive. If lipoproteins were isolated from the blood of donors, there might be risks of disease transmission. Bacterial expression of the lipoprotein or apolipoprotein might be an effective approach to avoid disease transmission, although it is expensive. Basing a platform on a peptide mimetic of apolipoproteins may also be an advantageous approach, since such peptides can be easily synthesized in pathogen-free, GMP processes. Scalable and commercially viable synthesis will have to be developed. Despite the natural origins of any selected agent, safety will have to be proven via in vitro and animals experiments prior to clinical trials. However, given the implementation of clinical trials of reconstituted HDL as antiatherosclerosis therapies,[216] it should be possible to find practical paths to clinical trials for lipoprotein delivery agents. The most likely next lipoprotein based agents to enter the clinic are lipoproteins loaded with already approved drugs such as HDL loaded with simvastatin or LDL loaded with doxorubicin. The known biocompatibility (or tolerability) of the individual components would de-risk a novel therapy based on their combination, encouraging investment and lowering FDA concerns. Clinical translation of lipoproteins delivering novel drug molecules, nucleic acids, fluorophores or biodegradable nanocrystals such as iron oxides is likely somewhat further down the road due to the need for further research to optimize and/or conduct extensive toxicological assessments. Testing in man of lipoproteins labeled with gadolinium seems least likely at this time, due to the concerns over the adverse health effects caused by gadolinium retention such as nephrogenic systemic fibrosis.[217, 218] 


\section{Conclusions}

Lipoproteins have proven themselves to be effective and versatile delivery agents. They can be modified in numerous ways, such as loading with drugs, paramagnetic lipids, fluorophores, nanocrystals, radioactive nuclei, boron compounds and nucleic acids. Lipoproteins have a number of natural targets, while addition of ligands results in re-routing to other targets. These attributes have led to applications in imaging and therapy of cardiovascular diseases, cancer and Alzheimer's. Lipoproteins have certain inherent advantages over other delivery vehicles, such as biodegradability, biocompatibility and a lack of immunogenicity. Furthermore, imaging and studying lipoprotein behavior in vivo is of fundamental interest due to their important roles in biology. Tremendous innovation has been made in this technology over the last decade and we expect to see significant progress in the coming years.

\section{Acknowledgements}

This work was supported by NIH grants R01HL131557, R00EB012165 and R03CA171661, as well as by start-up funding from the University of Pennsylvania (all D.P.C.). J.S.R. thanks the National Institutes of Health and National Heart, Lung, and Blood Institute for a Vascular Surgery Scientist Training Program grant (T32HL094293). C.S.T. thanks the Howard Hughes Medical Institute (HHMI) for a Physician Scientist Early Career Award, the Department of Defense/Air Force Office of Scientific Research (FA95501310192), and the National Institutes of Health/National Cancer Institute (U54CA151880 and R01CA167041). 


\section{References}

[1] M.F. Kircher, J.K. Willmann, Molecular Body Imaging: MR Imaging, CT, and US. Part I. Principles, Radiology, 263 (2012) 633-643.

[2] D.R. Arifin, D.A. Kedziorek, Y. Fu, K.W.Y. Chan, M.T. McMahon, C.R. Weiss, D.L. Kraitchman, J.W.M. Bulte, Microencapsulated cell tracking, NMR Biomed., 26 (2013) $850-859$.

[3] D.P. Cormode, P. Naha, Z.A. Fayad, Nanoparticle contrast agents for computed tomography: A focus on micelles, Contrast Media Mol. Imaging, 9 (2014) 37-52.

[4] A.J. Mieszawska, W.J.M. Mulder, Z.A. Fayad, D.P. Cormode, Multifunctional gold nanoparticles for diagnosis and therapy of disease, Mol. Pharmaceutics, 10 (2013) 831847.

[5] M.F. Kircher, A. de la Zerda, J.V. Jokerst, C.L. Zavaleta, P.J. Kempen, E. Mittra, K. Pitter, R. Huang, C. Campos, F. Habte, R. Sinclair, C.W. Brennan, I.K. Mellinghoff, E.C. Holland, S.S. Gambhir, A brain tumor molecular imaging strategy using a new triplemodality MRI-photoacoustic-Raman nanoparticle, Nat. Med., 18 (2012) 829-834.

[6] D.P. Cormode, T. Skajaa, Z.A. Fayad, W.J.M. Mulder, Nanotechnology in medical imaging: probe design and applications, Arterioscler. Thromb. Vasc. Biol., 29 (2009) 992-1000.

[7] D. Peer, J.M. Karp, S. Hong, O.C. Farokhzad, R. Margalit, R. Langer, Nanocarriers as an emerging platform for cancer therapy, Nat. Nanotech., 2 (2007) 751-760.

[8] S. Svenson, Clinical translation of nanomedicines, Curr. Opin. Solid St. M., 16 (2012) 287-294.

[9] M. Patrick, Abraxane Sales Are Lower than Expected in 3Q15, 2015. 
[10] A. Gianella, P.A. Jarzyna, V. Mani, S. Ramachandran, C. Calcagno, J. Tang, B. Kann, W.J. Dijk, V.L. Thijssen, A.W. Griffioen, G. Storm, Z.A. Fayad, W.J. Mulder, Multifunctional nanoemulsion platform for imaging guided therapy evaluated in experimental cancer, ACS Nano, 5 (2011) 4422-4433.

[11] T.M. Allen, P.R. Cullis, Liposomal drug delivery systems: From concept to clinical applications, Adv. Drug Deliv. Rev., 65 (2013) 36-48.

[12] V.P. Torchilin, Micellar nanocarriers: Pharmaceutical perspectives, Pharmaceut. Res., 24 (2007) 1-16.

[13] W.T. Al-Jamal, K. Kostarelos, Liposomes: from a clinically established drug delivery system to a nanoparticle platform for theranostic nanomedicine, Acc. Chem. Res., 44 (2011) 1094-1104.

[14] M. Elsabahy, K.L. Wooley, Design of polymeric nanoparticles for biomedical delivery applications, Chem. Soc. Rev., 41 (2012) 2545-2561.

[15] L. Brannon-Peppas, J.O. Blanchette, Nanoparticle and targeted systems for cancer therapy, Adv. Drug Deliv. Rev., 64 (2012) 206-212.

[16] L.F. Zhang, J.M. Chan, F.X. Gu, J.W. Rhee, A.Z. Wang, A.F. Radovic-Moreno, F. Alexis, R. Langer, O.C. Farokhzad, Self-assembled lipid-polymer hybrid nanoparticles: A robust drug delivery platform, ACS Nano, 2 (2008) 1696-1702.

[17] B. Mandal, H. Bhattacharjee, N. Mittal, H. Sah, P. Balabathula, L.A. Thoma, G.C. Wood, Core-shell-type lipid-polymer hybrid nanoparticles as a drug delivery platform, Nanomedicine: NBM, 9 (2013) 474-491.

[18] J. Khandare, M. Calderon, N.M. Dagia, R. Haag, Multifunctional dendritic polymers in nanomedicine: opportunities and challenges, Chem. Soc. Rev., 41 (2012) 2824-2848. 
[19] M. Balcioglu, M. Rana, M.V. Yigit, Doxorubicin loading on graphene oxide, iron oxide and gold nanoparticle hybrid, J. Mater. Chem. B, 1 (2013) 6187-6193.

[20] J. Liu, L. Cui, D. Losic, Graphene and graphene oxide as new nanocarriers for drug delivery applications, Acta Biomater., 9 (2013) 9243-9257.

[21] S.K. Libutti, G.F. Paciotti, A.A. Byrnes, H.R. Alexander, W.E. Gannon, M. Walker, G.D. Seidel, N. Yuldasheva, L. Tamarkin, Phase I and pharmacokinetic studies of CYT6091, a novel PEGylated colloidal gold-rhTNF nanomedicine, Clin. Cancer Res., 16 (2010) 6139-6149.

[22] C. Tassa, S.Y. Shaw, R. Weissleder, Dextran-coated iron oxide nanoparticles: a versatile platform for targeted molecular imaging, molecular diagnostics, and therapy, Acc. Chem. Res., 44 (2011) 842-852.

[23] S.M.C. Berman, P. Walczak, J.W.M. Bulte, Tracking stem cells using magnetic nanoparticles, Wiley Interdiscip. Rev. Nanomed. Nanobiotechnol., 3 (2011) 343-355. [24] N. Lee, T. Hyeon, Designed synthesis of uniformly sized iron oxide nanoparticles for efficient magnetic resonance imaging contrast agents, Chem. Soc. Rev., 41 (2012) 2575-2589.

[25] P. Naha, A. Al-Zaki, E.R. Hecht, M. Chorny, P. Chhour, E. Blankemeyer, D.M. Yates, W.R.T. Witschey, H.I. Litt, A. Tsourkas, D.P. Cormode, Dextran coated bismuthiron oxide nanohybrid contrast agents for computed tomography and magnetic resonance imaging, J. Mater. Chem. B, 2 (2014) 8239-8248.

[26] Z.X. Li, J.C. Barnes, A. Bosoy, J.F. Stoddart, J.I. Zink, Mesoporous silica nanoparticles in biomedical applications, Chem. Soc. Rev., 41 (2012) 2590-2605. 
[27] J.L. Vivero-Escoto, R.C. Huxford-Phillips, W.B. Lin, Silica-based nanoprobes for biomedical imaging and theranostic applications, Chem. Soc. Rev., 41 (2012) 2673-2685. [28] D.P. Cormode, P.A. Jarzyna, W.J.M. Mulder, Z.A. Fayad, Modified natural nanoparticles as contrast agents for medical imaging, Adv. Drug Deliv. Rev., 62 (2010) 329-338.

[29] T. Skajaa, D.P. Cormode, E. Falk, W.J.M. Mulder, E.A. Fisher, Z.A. Fayad, High density lipoprotein-based contrast agents for multimodal imaging of atherosclerosis, Arterioscler. Thromb. Vasc. Biol., 30 (2010) 169-176.

[30] M.G. Damiano, R.K. Mutharasan, S. Tripathy, K.M. McMahon, C.S. Thaxton, Templated high density lipoprotein nanoparticles as potential therapies and for molecular delivery, Adv. Drug Deliv. Rev., 65 (2013) 649-662.

[31] H. Huang, W. Cruz, J. Chen, G. Zheng, Learning from biology: synthetic lipoproteins for drug delivery, Wiley Interdiscip. Rev. Nanomed. Nanobiotechnol., 7 (2015) 298-314.

[32] R. Kannan Mutharasan, L. Foit, C. Shad Thaxton, High-density lipoproteins for therapeutic delivery systems, J. Mater. Chem. B, 4 (2016) 188-197.

[33] B. Schwarz, T. Douglas, Development of virus-like particles for diagnostic and prophylactic biomedical applications, Wiley Interdiscip. Rev. Nanomed. Nanobiotechnol., 7 (2015) 722-735.

[34] K.J. Koudelka, M. Manchester, Chemically modified viruses: principles and applications, Curr. Opin. Chem. Biol., 14 (2010) 810-817. 
[35] K.M. Bennett, H. Zhou, J.P. Sumner, S.J. Dodd, N. Bouraoud, K. Doi, R.A. Star, A.P. Koretsky, MRI of the basement membrane using charged nanoparticles as contrast agents, Magn. Reson. Med., 60 (2008) 564-574.

[36] Z.P. Zhen, W. Tang, T. Todd, J. Xie, Ferritins as nanoplatforms for imaging and drug delivery, Expert Opin. Drug Deliv., 11 (2014) 1913-1922.

[37] B. Bhushan, S.U. Kumar, I. Matai, A. Sachdev, P. Dubey, P. Gopinath, Ferritin nanocages: A novel platform for biomedical applications, J. Biomed. Nanotech., 10 (2014) 2950-2976.

[38] D.C. Buehler, M.D. Marsden, S. Shen, D.B. Toso, X. Wu, J.A. Loo, Z.H. Zhou, V.A. Kickhoefer, P.A. Wender, J.A. Zack, L.H. Rome, Bioengineered vaults: Selfassembling protein shell-lipophilic core nanoparticles for drug delivery, ACS Nano, 8 (2014) 7723-7732.

[39] I.E. Allijn, W. Leong, J. Tang, A. Gianella, A.J. Mieszawska, F. Fay, G. Ma, S. Russell, C.B. Callo, R.E. Gordon, E. Korkmaz, J.A. Post, Y. Zhao, H.C. Gerritsen, G. Storm, A. Thran, R. Proksa, H. Daerr, V. Fuster, E.A. Fisher, Z.A. Fayad, W.J. Mulder, D.P. Cormode, Gold nanocrystal labeling allows low density lipoprotein imaging from the subcellular to macroscopic level, ACS Nano, 7 (2013) 9761-9770.

[40] D.P. Cormode, T. Skajaa, M.M. van Schooneveld, R. Koole, P. Jarzyna, M.E. Lobatto, C. Calcagno, A. Barazza, R.E. Gordon, P. Zanzonico, E.A. Fisher, Z.A. Fayad, W.J.M. Mulder, Nanocrystal core high-density lipoproteins: A multimodal molecular imaging contrast agent platform, Nano Lett., 8 (2008) 3715-3723. 
[41] K.C. Vickers, P. Sethupathy, J. Baran-Gale, A.T. Remaley, Complexity of microRNA function and the role of isomiRs in lipid homeostasis, J. Lipid Res., 54 (2013) 1182-1191.

[42] W. Chen, P.A. Jarzyna, G.A.F. van Tilborg, V.A. Nguyen, D.P. Cormode, A. Klink, A.W. Griffioen, G.J. Randolph, E.A. Fisher, W.J.M. Mulder, Z.A. Fayad, RGD-Peptide functionalized and reconstituted high density lipoprotein nanoparticles as a versatile and multimodal tumor targeting molecular imaging probe, FASEB J., 24 (2010) 1689-1699. [43] G. Zheng, J. Chen, H. Li, J.D. Glickson, Rerouting lipoprotein nanoparticles to selected alternate receptors for the targeted delivery of cancer diagnostic and therapeutic agents, Proc. Natl. Acad. Sci. USA, 102 (2005) 17757-17762.

[44] R. Duivenvoorden, J. Tang, D.P. Cormode, A.J. Mieszawska, D. Izquierdo-Garcia, C. Ozcan, M.J. Otten, N. Zaidi, M.E. Lobatto, S.M. van Rijs, B. Priem, E.L. Kuan, C. Martel, B. Hewing, H. Sager, M. Nahrendorf, G.J. Randolph, E.S.G. Stroes, V. Fuster, E.A. Fisher, Z.A. Fayad, W.J.M. Mulder, A statin-loaded reconstituted high-density lipoprotein nanoparticle as a targeted therapy for atherosclerotic plaque inflammation, Nat. Commun., 5 (2014) 3065.

[45] L. Song, H. Li, U. Sunar, J. Chen, I. Corbin, A. Yodh, G. Zheng, Naphthalocyaninereconstituted LDL nanoparticles for in vivo cancer imaging and treatment, Int. J. Nanomedicine, 2 (2007) 767-774.

[46] M.E. Davis, Z.G. Chen, D.M. Shin, Nanoparticle therapeutics: an emerging treatment modality for cancer, Nat. Rev. Drug Discov., 7 (2008) 771-782.

[47] S. Eisenberg, H.G. Windmueller, R.I. Levy, Metabolic fate of rat and human lipoprotein apoproteins in the rat, J. Lipid Res., 14 (1973) 446-458. 
[48] J.P. Segrest, D.W. Garber, C.G. Brouillette, S.C. Harvey, G.M. Anantharamaiah, The amphipathic alpha helix: a multifunctional structural motif in plasma apolipoproteins, Adv. Protein Chem., 45 (1994) 303-369.

[49] R.J. Havel, H.A. Eder, J.H. Bragdon, The distribution and chemical composition of ultracentrifugally separated lipoproteins in human serum, J. Clin. Invest., 34 (1955) 13451353

[50] D.A. Bricarello, J.T. Smilowitz, A.M. Zivkovic, J.B. German, A.N. Parikh, Reconstituted lipoprotein: A versatile class of biologically inspired nanostructures, ACS Nano, 5 (2011) 42-57.

[51] A.V. Nichols, R.M. Krauss, T.A. Musliner, Nondenaturing polyacrylamide gradient gel electrophoresis, Method Enzymol., 128 (1986) 417-431.

[52] J.A. Yáñez, S.W.J. Wang, I.W. Knemeyer, M.A. Wirth, K.B. Alton, Intestinal lymphatic transport for drug delivery, Adv. Drug Deliv. Rev., 63 (2011) 923-942. [53] E. de Faria, L.G. Fong, M. Komaromy, A.D. Cooper, Relative roles of the LDL receptor, the LDL receptor-like protein, and hepatic lipase in chylomicron remnant removal by the liver, J. Lipid Res., 37 (1996) 197-209.

[54] G.S. Shelness, J.A. Sellers, Very-low-density lipoprotein assembly and secretion, Curr. Opin. Lipidol., 12 (2001) 151-157.

[55] M. Merkel, R.H. Eckel, I.J. Goldberg, Lipoprotein lipase: genetics, lipid uptake, and regulation, J. Lipid Res., 43 (2002) 1997-2006.

[56] R.A. Hegele, Plasma lipoproteins: genetic influences and clinical implications, Nat. Rev. Genet., 10 (2009) 109-121. 
[57] I. Ramasamy, Recent advances in physiological lipoprotein metabolism, Clin. Chem. Lab. Med., 52 (2014) 1695-1727.

[58] J. Babiak, L.L. Rudel, Lipoproteins and atherosclerosis, Baillieres Clin. Endocrinol. Metab., 1 (1987) 515-550.

[59] R.S. Rosenson, H.B. Brewer, Jr., M.J. Chapman, S. Fazio, M.M. Hussain, A.

Kontush, R.M. Krauss, J.D. Otvos, A.T. Remaley, E.J. Schaefer, HDL measures, particle heterogeneity, proposed nomenclature, and relation to atherosclerotic cardiovascular events, Clin Chem, 57 (2011) 392-410.

[60] S. Acton, A. Rigotti, K.T. Landschulz, S. Xu, H.H. Hobbs, M. Kriegert, Identification of scavenger receptor SR-BI as a high density lipoprotein receptor, Science, 271 (1996) 518-520.

[61] A. Kontush, M. Lhomme, M.J. Chapman, Unraveling the complexities of the HDL lipidome, J. Lipid Res., 54 (2013) 2950-2963.

[62] A.S. Shah, L. Tan, J.L. Long, W.S. Davidson, Proteomic diversity of high density lipoproteins: our emerging understanding of its importance in lipid transport and beyond, J. Lipid Res., 54 (2013) 2575-2585.

[63] M. Naghavi, P. Libby, E. Falk, S.W. Casscells, S. Litovsky, J. Rumberger, J.J.

Badimon, C. Stefanadis, P. Moreno, G. Pasterkamp, Z. Fayad, P.H. Stone, S. Waxman, P. Raggi, M. Madjid, A. Zarrabi, A. Burke, C. Yuan, P.J. Fitzgerald, D.S. Siscovick, C.L. de Korte, M. Aikawa, K.E.J. Airaksinen, G. Assmann, C.R. Becker, J.H. Chesebro, A. Farb, Z.S. Galis, C. Jackson, I.K. Jang, W. Koenig, R.A. Lodder, K. March, J. Demirovic, M. Navab, S.G. Priori, M.D. Rekhter, R. Bahr, S.M. Grundy, R. Mehran, A. Colombo, E. Boerwinkle, C. Ballantyne, W. Insull, R.S. Schwartz, R. Vogel, P.W. Serruys, G.K. 
Hansson, D.P. Faxon, S. Kaul, H. Drexler, P. Greenland, J.E. Muller, R. Virmani, P.M. Ridker, D.P. Zipes, P.K. Shah, J.T. Willerson, From vulnerable plaque to vulnerable patient - A call for new definitions and risk assessment strategies: Part I, Circulation, 108 (2003) 1664-1672.

[64] J. Shepherd, S.M. Cobbe, I. Ford, C.G. Isles, A.R. Lorimer, P.W. Macfarlane, J.H. McKillop, C.J. Packard, Prevention of coronary heart disease with pravastatin in men with hypercholesterolemia, New Eng. J. Med., 333 (1995) 1301-1307.

[65] Therapeutic response to lovastatin (mevinolin) in nonfamilial hypercholesterolemia. A multicenter study. The Lovastatin Study Group II, JAMA, 256 (1986) 2829-2834.

[66] D.W. Bilheimer, S.M. Grundy, M.S. Brown, J.L. Goldstein, Mevinolin and colestipol stimulate receptor-mediated clearance of low density lipoprotein from plasma in familial hypercholesterolemia heterozygotes, Proc Natl Acad Sci U S A, 80 (1983) $4124-4128$.

[67] K.J. Moore, I.J. Goldberg, Emerging roles of PCSK9: More than a one-trick pony, Arterioscler. Thromb. Vasc. Biol., 36 (2016) 211-212.

[68] D.J. Gordon, B.M. Rifkind, High-density lipoprotein - the clinical implications of recent studies, New Eng. J Med., 321 (1989) 1311-1316.

[69] G.G. Schwartz, A.G. Olsson, M. Abt, C.M. Ballantyne, P.J. Barter, J. Brumm, B.R. Chaitman, I.M. Holme, D. Kallend, L.A. Leiter, E. Leitersdorf, J.J.V. McMurray, H. Mundl, S.J. Nicholls, P.K. Shah, J.-C. Tardif, R.S. Wright, Effects of dalcetrapib in patients with a recent acute coronary syndrome, New Eng. J. Med., 367 (2012) 20892099. 
[70] J.A. Glomset, J.L. Wright, Some properties of a cholesterol esterifying enzyme in human plasma, Biochim. Biophys. Acta, 89 (1964) 266-276.

[71] G.H. Rothblat, M.C. Phillips, High-density lipoprotein heterogeneity and function in reverse cholesterol transport, Curr. Opin. Lipidol., 21 (2010) 229-238.

[72] R. Firestone, Low-density lipoprotein as a vehicle for targeting antitumor compounds to cancer cells, Bioconjugate Chem., 5 (1994) 105-113.

[73] R.S. Rosenson, H.B. Brewer, W.S. Davidson, Z.A. Fayad, V. Fuster, J. Goldstein, M. Hellerstein, X.C. Jiang, M.C. Phillips, D.J. Rader, A.T. Remaley, G.H. Rothblat, A.R. Tall, L. Yvan-Charvet, Cholesterol efflux and atheroprotection advancing the concept of reverse cholesterol transport, Circulation, 125 (2012) 1905-1919.

[74] N. Sabnis, M. Nair, M. Israel, W.J. McConathy, A.G. Lacko, Enhanced solubility and functionality of valrubicin (AD-32) against cancer cells upon encapsulation into biocompatible nanoparticles, Int. J. Nanomedicine, 7 (2012) 975-983.

[75] B.A. Kingwell, M.J. Chapman, A. Kontush, N.E. Miller, HDL-targeted therapies: progress, failures and future, Nat. Rev. Drug Discov., 13 (2014) 445-464.

[76] B.R. Krause, A.T. Remaley, Reconstituted HDL for the acute treatment of acute coronary syndrome, Curr. Opin. Lipidol., 24 (2013) 480-486.

[77] G. Franceschini, C.R. Sirtori, A. Capurso, 2nd, K.H. Weisgraber, R.W. Mahley, A-I Milano apoprotein. Decreased high density lipoprotein cholesterol levels with significant lipoprotein modifications and without clinical atherosclerosis in an Italian family, J. Clin. Invest., 66 (1980) 892-900.

[78] S.E. Nissen, T. Tsunoda, E.M. Tuzcu, P. Schoenhagen, C.J. Cooper, M. Yasin, G.M. Eaton, M.A. Lauer, W.S. Sheldon, C.L. Grines, S. Halpern, T. Crowe, J.C. Blankenship, 
R. Kerensky, Effect of recombinant ApoA-I Milano on coronary atherosclerosis in patients with acute coronary syndromes: a randomized controlled trial, JAMA, 290 (2003) 2292-2300.

[79] S.J. Nicholls, K. Uno, Y. Kataoka, S.E. Nissen, ETC-216 for coronary artery disease, Expert Opin. Biol. Ther., 11 (2011) 387-394.

[80] P.G. Lerch, V. Fortsch, G. Hodler, R. Bolli, Production and characterization of a reconstituted high density lipoprotein for therapeutic applications, Vox Sang, 71 (1996) 155-164.

[81] J.C. Tardif, J. Gregoire, P.L. L'Allier, R. Ibrahim, J. Lesperance, T.M. Heinonen, S. Kouz, C. Berry, R. Basser, M.A. Lavoie, M.C. Guertin, J. Rodes-Cabau, H.D.L.o.A.-S. Effect of r, I. Efficacy, Effects of reconstituted high-density lipoprotein infusions on coronary atherosclerosis: a randomized controlled trial, JAMA, 297 (2007) 1675-1682. [82] R. Easton, A. Gille, D. D'Andrea, R. Davis, S.D. Wright, C. Shear, A multiple ascending dose study of CSL112, an infused formulation of ApoA-I, J. Clin. Pharmacol., 54 (2014) 301-310.

[83] A. Gille, R. Easton, D. D'Andrea, S.D. Wright, C.L. Shear, CSL112 enhances biomarkers of reverse cholesterol transport after single and multiple infusions in healthy subjects, Arterioscler. Thromb. Vasc. Biol., 34 (2014) 2106-2114.

[84] A.C. Calkin, B.G. Drew, A. Ono, S.J. Duffy, M.V. Gordon, S.M. Schoenwaelder, D. Sviridov, M.E. Cooper, B.A. Kingwell, S.P. Jackson, Reconstituted high-density lipoprotein attenuates platelet function in individuals with type 2 diabetes mellitus by promoting cholesterol efflux, Circulation, 120 (2009) 2095-2104. 
[85] J.C. Tardif, C.M. Ballantyne, P. Barter, J.L. Dasseux, Z.A. Fayad, M.C. Guertin, J.J. Kastelein, C. Keyserling, H. Klepp, W. Koenig, P.L. L'Allier, J. Lesperance, T.F. Luscher, J.F. Paolini, A. Tawakol, D.D. Waters, C.H.D.L.I.S.Q.A.R. Investigators, Effects of the high-density lipoprotein mimetic agent CER-001 on coronary atherosclerosis in patients with acute coronary syndromes: a randomized trial, Eur. Heart J., 35 (2014) 3277-3286.

[86] G.K. Hovingh, L.P. Smits, C. Stefanutti, H. Soran, S. Kwok, J. de Graaf, D. Gaudet, C.H. Keyserling, H. Klepp, J. Frick, J.F. Paolini, J.L. Dasseux, J.J. Kastelein, E.S. Stroes, The effect of an apolipoprotein A-I-containing high-density lipoprotein-mimetic particle (CER-001) on carotid artery wall thickness in patients with homozygous familial hypercholesterolemia: The Modifying Orphan Disease Evaluation (MODE) study, Am. Heart J., 169 (2015) 736-742 e731.

[87] P.C.N. Rensen, R.L.A. de Vrueh, J. Kuiper, M.K. Bijsterbosch, E.A.L. Biessen, T.J.C. van Berkel, Recombinant lipoproteins: lipoprotein-like lipid particles for drug targeting, Adv. Drug Deliv. Rev., 47 (2001) 251-276.

[88] S. Geninatti Crich, S. Lanzardo, D. Alberti, S. Belfiore, A. Ciampa, G.B.

Giovenzana, C. Lovazzano, R. Pagliarin, S. Aime, Magnetic resonance imaging detection of tumor cells by targeting low-density lipoprotein receptors with Gd-loaded low-density lipoprotein particles, Neoplasia, 9 (2007) 1046-1056.

[89] N.O. Fischer, D.R. Weilhammer, A. Dunkle, C. Thomas, M. Hwang, M. Corzett, C. Lychak, W. Mayer, S. Urbin, N. Collette, J. Chiun Chang, G.G. Loots, A. Rasley, C.D. Blanchette, Evaluation of nanolipoprotein particles (NLPs) as an in vivo delivery platform, PLoS ONE, 9 (2014) e93342. 
[90] W. Chen, D.P. Cormode, Y. Vengrenyuk, B. Herranz, F.J. E., A. Klink, W.J.M. Mulder, E.A. Fisher, Z.A. Fayad, Collagen-specific peptide conjugated high density lipoprotein nanoparticles as MRI contrast agents to evaluate the stabilization of atherosclerotic plaque under regression conditions, JACC: Cardiovascular Imaging, 6 (2013) 373-384.

[91] J.C. Frias, K.J. Williams, E.A. Fisher, Z.A. Fayad, Recombinant HDL-like nanoparticles: a specific contrast agent for MRI of atherosclerotic plaques, J. Am. Chem. Soc., 126 (2004) 16316-16317.

[92] K.M. McMahon, R.K. Mutharasan, S. Tripathy, D. Veliceasa, M. Bobeica, D.K. Shumaker, A.J. Luthi, B.T. Helfand, H. Ardehali, C.A. Mirkin, O. Volpert, C.S. Thaxton, Biomimetic high density lipoprotein nanoparticles for nucleic acid delivery, Nano Lett., $11(2011)$ 1208-1214.

[93] Q. Song, M. Huang, L. Yao, X. Wang, X. Gu, J. Chen, J. Chen, J. Huang, Q. Hu, T. Kang, Z. Rong, H. Qi, G. Zheng, H. Chen, X. Gao, Lipoprotein-based nanoparticles rescue the memory loss of mice with Alzheimer's disease by accelerating the clearance of amyloid-beta, ACS Nano, 8 (2014) 2345-2359.

[94] J.C. Frias, Y. Ma, K.J. Williams, Z.A. Fayad, E.A. Fisher, Properties of a versatile nanoparticle platform contrast agent to image and characterize atherosclerotic plaques by magnetic resonance imaging, Nano Lett., 6 (2006) 2220-2224.

[95] C. Perez-Medina, J. Tang, D. Abdel-Atti, B. Hogstad, M. Merad, E.A. Fisher, Z.A. Fayad, J.S. Lewis, W.J. Mulder, T. Reiner, PET imaging of tumor-associated macrophages with 89Zr-labeled high-density lipoprotein nanoparticles, J. Nucl. Med., 56 (2015) 1272-1277. 
[96] D.P. Cormode, E. Roessl, A. Thran, T. Skajaa, R.E. Gordon, J.P. Schlomka, V. Fuster, E.A. Fisher, W.J.M. Mulder, R. Proksa, Z.A. Fayad, Atherosclerotic plaque composition: analysis with multicolor CT and targeted gold nanoparticles, Radiology, $256(2010) 774-782$.

[97] M. Navab, G.M. Anantharamaiah, S.T. Reddy, A.M. Fogelman, Apolipoprotein A-I mimetic peptides and their role in atherosclerosis prevention, Nat. Clin. Pract., Cardiovasc. Med., 3 (2006) 540-547.

[98] D.P. Cormode, K.C. Briley-Saebo, W.J.M. Mulder, J.G.S. Aguinaldo, A. Barazza, Y. Ma, E.A. Fisher, Z.A. Fayad, An apoA-I mimetic peptide HDL-based MRI contrast agent for atherosclerotic plaque composition detection, Small., 4 (2008) 1437-1444. [99] Z. Zhang, W. Cao, H. Jin, J.F. Lovell, M. Yang, L. Ding, J. Chen, I. Corbin, Q. Luo, G. Zheng, Biomimetic nanocarrier for direct cytosolic drug delivery, Angew. Chem., 48 (2009) 9171-9175.

[100] B.L. Sanchez-Gaytan, F. Fay, M.E. Lobatto, J. Tang, M. Ouimet, Y. Kim, S.E. van der Staay, S.M. van Rijs, B. Priem, L. Zhang, E.A. Fisher, K.J. Moore, R. Langer, Z.A. Fayad, W.J. Mulder, HDL-mimetic PLGA nanoparticle to target atherosclerosis plaque macrophages, Bioconjugate Chem, 26 (2015) 443-451.

[101] M. Nikanjam, E.A. Blakely, K.A. Bjornstad, X. Shu, T.F. Budinger, T.M. Forte, Synthetic nano-low density lipoprotein as targeted drug delivery vehicle for glioblastoma multiforme, Int. J. Pharm., 328 (2006) 86-94.

[102] D.P. Cormode, E.A. Fisher, E.S. Stroes, W.J. Mulder, Z.A. Fayad, High-density lipoprotein is a nanoparticle, but not all nanoparticles are high-density lipoprotein, Proc. Nat. Acad. Sci. USA, 110 (2013) E3548. 
[103] A. Shaish, G. Keren, P. Chouraqui, H. Levkovitz, D. Harats, Imaging of aortic atherosclerotic lesions by 125I-LDL, 125I-Oxidized-LDL, 125I-HDL and 125I-BSA, Pathobiology, 69 (2001) 225-229.

[104] D.P. Cormode, R. Chandrasekar, A. Delshad, K.C. Briley-Saebo, C. Calcagno, A. Barazza, W.J.M. Mulder, E.A. Fisher, Z.A. Fayad, Comparison of synthetic HDL contrast agents for MR imaging of atherosclerosis, Bioconjugate Chem., 20 (2009) 937,Äì943.

[105] T. Skajaa, D.P. Cormode, P.A. Jarzyna, A. Delshad, C. Blachford, A. Barazza, E.A. Fisher, R.E. Gordon, Z.A. Fayad, W.J.M. Mulder, The biological properties of iron oxide core high-density lipoprotein in experimental atherosclerosis, Biomaterials, 32 (2011) 206-213.

[106] T. Skajaa, Y. Zhao, D.J. van den Heuvel, H.C. Gerritsen, D.P. Cormode, R. Koole, M.M. van Schooneveld, J.A. Post, E.A. Fisher, Z.A. Fayad, C. de Mello Donega, W.J.M. Mulder, Quantum dot and Cy5.5 labeled nanoparticles to investigate lipoprotein biointeractions via Förster resonance energy transfer, Nano Lett., 10 (2010) 5131-5138. [107] Y. Kim, F. Fay, D.P. Cormode, B.L. Sanchez-Gaytan, J. Tang, E.J. Hennessy, M. Ma, K. Moore, O.C. Farokhzad, E.A. Fisher, W.J. Mulder, R. Langer, Z.A. Fayad, Single step reconstitution of multifunctional high-density lipoprotein-derived nanomaterials using microfluidics, ACS Nano, 7 (2013) 9975-9983.

[108] K.C. Briley-Saebo, S. Geninatti Crich, D.P. Cormode, A. Barazza, W.J.M. Mulder, W. Chen, G.B. Giovenzana, E.A. Fisher, S. Aime, Z.A. Fayad, High-relaxivity gadolinium-modified high-density lipoproteins as magnetic resonance imaging contrast agents, J. Phys. Chem. B, 113 (2009) 6283-6289 
[109] W. Chen, E. Vucic, E. Leupold, W.J.M. Mulder, D.P. Cormode, K.C. Briley-Saebo, A. Barazza, E.A. Fisher, M. Dathe, Z.A. Fayad, Incorporation of an apoE-derived lipopeptide in high-density lipoprotein MRI contrast agents for enhanced imaging of macrophages in atherosclerosis., Contrast Media Mol. Imaging, 3 (2008) 233-242. [110] C.E. Carney, I.L. Lenov, C.J. Baker, K.W. MacRenaris, A.L. Eckermann, S.G. Sligar, T.J. Meade, Nanodiscs as a modular platform for multimodal MR-optical imaging, Bioconjugate Chem., 26 (2015) 899-905.

[111] A.B. Sigalov, Nature-inspired nanoformulations for contrast-enhanced in vivo MR imaging of macrophages, Contrast Media Mol. Imaging, 9 (2014) 372-382.

[112] J.O. Lagerstedt, J. Petrlova, S. Hilt, A. Marek, Y. Chung, R. Sriram, M.S.

Budamagunta, J.F. Desreux, D. Thonon, T. Jue, A.I. Smirnov, J.C. Voss, EPR assessment of protein sites for incorporation of Gd(III) MRI contrast labels, Contrast Media Mol. Imaging, 8 (2013) 252-264.

[113] M. Rui, W. Guo, Q. Ding, X. Wei, J. Xu, Y. Xu, Recombinant high-density lipoprotein nanoparticles containing gadolinium-labeled cholesterol for morphologic and functional magnetic resonance imaging of the liver, Int. J. Nanomedicine, 7 (2012) 37513768.

[114] R. Sriram, J.O. Lagerstedt, J. Petrlova, H. Samardzic, U. Kreutzer, H. Xie, G.A. Kaysen, J.F. Desreux, D. Thonon, V. Jacques, M. Van Loane, J.C. Rutledge, M.N. Oda, J.C. Voss, T. Jue, Imaging apolipoprotein AI in vivo, NMR Biomed., 24 (2011) 916-924. [115] Q. Wang, S. Chen, Q. Luo, M. Liu, X. Zhou, A europium-lipoprotein nanocomposite for highly-sensitive MR-fluorescence multimodal imaging, RSC Adv., 5 (2015) 1808-1811. 
[116] W. Cao, K.K. Ng, I. Corbin, Z. Zhang, L. Ding, J. Chen, G. Zheng, Synthesis and evaluation of a stable bacteriochlorophyll-analog and its incorporation into high-density lipoprotein nanoparticles for tumor imaging, Bioconjugate Chem., 20 (2009) 2023-2031. [117] T. Ozawa, H. Yoshimura, S.B. Kim, Advances in fluorescence and bioluminescence imaging, Anal. Chem., 85 (2013) 590-609.

[118] C.R. White, D.W. Garber, G.M. Anantharamaiah, Anti-inflammatory and cholesterol-reducing properties of apolipoprotein mimetics: a review, J. Lipid Res., 55 (2014) 2007-2021.

[119] L. Chen, G. Yang, J. Monslow, L. Todd, D.P. Cormode, J. Tang, G.R. Grant, J.H. DeLong, S.Y. Tang, J.A. Lawson, E. Pure, G.A. FitzGerald, Myeloid cell microsomal prostaglandin E synthase-1 fosters atherogenesis in mice, Proc. Nat. Acad. Sci. USA, 111 (2014) 6828-6833.

[120] L. Cui, Q. Lin, C.S. Jin, W. Jiang, H. Huang, L. Ding, N. Muhanna, J.C. Irish, F. Wang, J. Chen, G. Zheng, A PEGylation-free biomimetic porphyrin nanoplatform for personalized cancer theranostics, ACS Nano, 9 (2015) 4484-4495.

[121] J. Rieffel, U. Chitgupi, J.F. Lovell, Recent advances in higher-order, multimodal, biomedical imaging agents, Small, 11 (2015) 4445-4461.

[122] I.R. Corbin, K.K. Ng, L. Ding, A. Jurisicova, G. Zheng, Near-infrared fluorescent imaging of metastatic ovarian cancer using folate receptor-targeted high-density lipoprotein nanocarriers, Nanomedicine (Lond), 8 (2013) 875-890.

[123] Z. Zhang, J. Chen, L. Ding, H. Jin, J.F. Lovell, I.R. Corbin, W. Cao, P.C. Lo, M. Yang, M.S. Tsao, Q. Luo, G. Zheng, HDL-mimicking peptide-lipid nanoparticles with improved tumor targeting, Small, 6 (2010) 430-437. 
[124] C. Jung, M.G. Kaul, O.T. Bruns, T. Ducic, B. Freund, M. Heine, R. Reimer, A. Meents, S.C. Salmen, H. Weller, P. Nielsen, G. Adam, J. Heeren, H. Ittrich, Intraperitoneal injection improves the uptake of nanoparticle-labeled high-density lipoprotein to atherosclerotic plaques compared with intravenous injection: a multimodal imaging study in ApoE knockout mice, Circ. Cardiovasc. Imaging, 7 (2014) 303-311. [125] J. Pietzsch, R. Bergmann, K. Rode, C. Hultsch, B. Pawelke, F. Wuest, J. van den Hoff, Fluorine-18 radiolabeling of low-density lipoproteins: a potential approach for characterization and differentiation of metabolism of native and oxidized low-density lipoproteins in vivo, Nucl. Med. Biol., 31 (2004) 1043-1050.

[126] J.M. Rosen, S.P. Butler, G.E. Meinken, T.S.T. Wang, R. Ramakrishnan, S.C. Srivastava, P.O. Alderson, H.N. Ginsberg, Indium-111-labeled LDL: a potential agent for imaging atherosclerotic disease and lipoprotein biodistribution, J. Nucl. Med., 31 (1990) 343-350.

[127] H.N. Ginsberg, S.J. Goldsmith, S. Vallabajosula, Noninvasive imaging of 99mTechnetium-labeled low density lipoprotein uptake by tendon xanthomas in hypercholesterolemic patients, Arteriosclerosis, 10 (1990) 256-262.

[128] H. Sinzinger, H. Bergmann, J. Kaliman, P. Angelberger, Imaging of human atherosclerosis lesions using I-123 low-density-lipoprotein, Eur. J. Nucl. Med., 12 (1986) 291-292.

[129] L. Iuliano, A. Signore, S. Vallabajosula, A.R. Colavita, C. Camastra, G. Ronga, C. Alessandri, E. Sbarigia, P. Fiorani, F. Violi, Preparation and biodistribution of (99m)technetium labelled oxidized LDL in man, Atherosclerosis, 126 (1996) 131-141. 
[130] G. Zheng, H. Li, M. Zhang, S. Lund-Katz, B. Chance, J.D. Glickson, Low-density lipoprotein reconstituted by pyropheophorbide cholesteryl oleate as target-specific photosensitizer, Bioconjugate Chem., 13 (2002) 392-396.

[131] S.P. Wu, I. Lee, P.P. Ghoroghchian, P.R. Frail, G. Zheng, J.D. Glickson, M.J. Therien, Near-infrared optical Imaging of B16 melanoma cells via low-density lipoprotein-mediated uptake and delivery of high emission dipole strength tris[(porphinato)zinc(II)] fluorophores, Bioconjugate Chem., 16 (2005) 542-550. [132] H. Li, Z. Zhang, D. Blessington, D.S. Nelson, R. Zhou, S. Lund-Katz, B. Chance, J.D. Glickson, G. Zheng, Carbocyanine labeled LDL for optical imaging of tumors, Acad. Radiol., 11 (2004) 669-677.

[133] L.M. Mitsumori, J.L. Ricks, M.E. Rosenfeld, U.P. Schmiedl, C. Yuan, Development of a lipoprotein based molecular imaging MR contrast agent for the noninvasive detection of early atherosclerotic disease, Int. J. Cardiovasc. Imaging, 20 (2004) 561-567.

[134] B. Drahoš, I. Lukeš, E. Tóth, Manganese(II) Complexes as Potential Contrast Agents for MRI, Eur. J. Inorg. Chem., 2012 (2012) 1975-1986.

[135] H. Li, B.D. Gray, I.R. Corbin, C. Lebherz, H. Choi, S. Lund-Katz, J.M. Wilson, J.D. Glickson, R. Zhou, MR and fluorescent imaging of low-density lipoprotein receptors, Acad. Radiol., 11 (2004) 1251-1259.

[136] I.R. Corbin, H. Li, J. Chen, S. Lund-Katz, R. Zhou, J.D. Glickson, G. Zheng, Lowdensity lipoprotein nanoparticles as magnetic resonance imaging contrast agents, Neoplasia, 8 (2006) 488-498. 
[137] A.N. Lowell, H. Qiao, T. Liu, T. Ishikawa, H. Zhang, S. Oriana, M. Wang, E. Ricciotti, G.A. FitzGerald, R. Zhou, Y. Yamakoshi, Functionalized low-density lipoprotein nanoparticles for in vivo enhancement of atherosclerosis on magnetic resonance images, Bioconjugate Chem., 23 (2012) 2313-2319.

[138] Y. Yamakoshi, H. Qiao, A.N. Lowell, M. Woods, B. Paulose, Y. Naka, H. Zhang, T. Liu, S. Lund-Katz, R. Zhou, LDL-based nanoparticles for contrast enhanced MRI of atheroplaques in mouse models, Chem. Commun., 47 (2011) 8835-8837.

[139] J. Chen, I.R. Corbin, H. Li, W. Cao, J.D. Glickson, G. Zheng, Ligand conjugated low-density lipoprotein nanoparticles for enhanced optical cancer imaging in vivo, J. Am. Chem. Soc., 129 (2007) 5798-5799.

[140] M.L. Hill, I.R. Corbin, R.B. Levitin, W. Cao, J.G. Mainprize, M.J. Yaffe, G. Zheng, In vitro assessment of poly-iodinated triglyceride reconstituted low-density lipoprotein: initial steps toward CT molecular imaging, Acad. Radiol., 17 (2010) 13591365.

[141] M. Krieger, Reconstitution of the hydrophobic core of low-density lipoprotein, Meth. Enzymol., 128 (1986) 608-613.

[142] S. Ishibashi, M.S. Brown, J.L. Goldstein, R.D. Gerard, R.E. Hammer, J. Herz, Hypercholesterolemia in low density lipoprotein receptor knockout mice and its reversal by adenovirus-mediated gene delivery, J. Clin. Invest., 92 (1993) 883-893.

[143] M. Huettinger, J.R. Corbett, W.J. Schneider, J.T. Willerson, M.S. Brown, J.L. Goldstein, Imaging of hepatic low-density lipoprotein receptors by radionuclide scintiscanning in vivo, Proc. Nat. Acad. Sci. USA, 81 (1984) 7599-7603. 
[144] A. Barazza, C. Blachford, O. Even-Or, V.A. Joaquin, K.C. Briley-Saeboe, W. Chen, X.-C. Jiang, W.J.M. Mulder, D.P. Cormode, Z.A. Fayad, E.A. Fisher, The complex fate in plasma of gadolinium incorporated into high-density lipoproteins used for magnetic imaging of atherosclerotic plaques, Bioconjugate Chem., 24 (2013) 1039-1048. [145] N. Xu, L. Zhou, R. Odselius, A. Nilsson, Uptake of radiolabeled and colloidal gold-labeled chyle chylomicrons and chylomicron remnants by rat platelets in vitro, Arterioscler. Thromb. Vasc. Biol., 15 (1995) 972-981.

[146] A. Bowler, T.G. Redgrave, J.C.L. Mamo, Chylomicron-remnant clearance in homozygote and heterozygote Watanabe-heritable-hyperlipidaemic rabbits is defective. Lack of evidence for an independent chylomicron-remnant receptor, Biochem. J., 276 (1991) 381-386.

[147] O.T. Bruns, H. Ittrich, K. Peldschus, M.G. Kaul, U.I. Tromsdorf, J. Lauterwasser, M.S. Nikolic, B. Mollwitz, M. Merkel, N.C. Bigall, S. Sapra, R. Reimer, H. Hohenberg, H. Weller, A. Eychmuller, G. Adam, U. Beisiegel, J. Heeren, Real-time magnetic resonance imaging and quantification of lipoprotein metabolism in vivo using nanocrystals, Nat. Nano., 4 (2009) 193-201.

[148] E. Huynh, G. Zheng, Engineering multifunctional nanoparticles: all-in-one versus one-for-all, Wiley Interdiscip. Rev. Nanomed. Nanobiotechnol., 5 (2013) 250-265.

[149] N. Sabnis, A.G. Lacko, Drug delivery via lipoprotein-based carriers: answering the challenges in systemic therapeutics, Ther. Deliv., 3 (2012) 599-608.

[150] T. Tanaka, S. Shiramoto, M. Miyashita, Y. Fujishima, Y. Kaneo, Tumor targeting based on the effect of enhanced permeability and retention (EPR) and the mechanism of receptor-mediated endocytosis (RME), Int. J. Pharm., 277 (2004) 39-61. 
[151] U. Prabhakar, H. Maeda, R.K. Jain, E.M. Sevick-Muraca, W. Zamboni, O.C.

Farokhzad, S.T. Barry, A. Gabizon, P. Grodzinski, D.C. Blakey, Challenges and key considerations of the enhanced permeability and retention effect for nanomedicine drug delivery in oncology, Cancer Res., 73 (2013) 2412-2417.

[152] A.L. Klibanov, K. Maruyama, A.M. Beckerleg, V.P. Torchilin, L. Huang, Activity of amphipathic poly(ethylene glycol)-5000 to prolong the circulation time of liposomes depends on the liposome size and is unfavorable for immunoliposome binding to target, Biochim. Biophys. Acta, 1062 (1991) 142-148.

[153] C. Danilo, J.L. Gutierrez-Pajares, M.A. Mainieri, I. Mercier, M.P. Lisanti, P.G. Frank, Scavenger receptor class B type I regulates cellular cholesterol metabolism and cell signaling associated with breast cancer development, Breast Cancer Res., 15 (2013) R87.

[154] A. Gorin, L. Gabitova, I. Astsaturov, Regulation of cholesterol biosynthesis and cancer signaling, Curr. Opin. Pharmacol., 12 (2012) 710-716.

[155] C.G. Leon, J.A. Locke, H.H. Adomat, S.L. Etinger, A.L. Twiddy, R.D. Neumann, C.C. Nelson, E.S. Guns, K.M. Wasan, Alterations in cholesterol regulation contribute to the production of intratumoral androgens during progression to castration-resistant prostate cancer in a mouse xenograft model, Prostate, 70 (2010) 390-400.

[156] G. Llaverias, C. Danilo, I. Mercier, K. Daumer, F. Capozza, T.M. Williams, F. Sotgia, M.P. Lisanti, P.G. Frank, Role of cholesterol in the development and progression of breast cancer, Am. J. Pathol., 178 (2011) 402-412. 
[157] S. Muntoni, L. Atzori, R. Mereu, G. Satta, M.D. Macis, M. Congia, A. Tedde, A. Desogus, S. Muntoni, Serum lipoproteins and cancer, Nutr. Metab. Cardiovasc. Dis., 19 (2009) 218-225.

[158] M.M.K. Shahzad, L.S. Mangala, H.D. Han, C.H. Lu, J. Bottsford-Miller, M. Nishimura, E.M. Mora, J.W. Lee, R.L. Stone, C.V. Pecot, D. Thanapprapasr, J.W. Roh, P. Gaur, M.P. Nair, Y.Y. Park, N. Sabnis, M.T. Deavers, J.S. Lee, L.M. Ellis, G. LopezBerestein, W.J. McConathy, L. Prokai, A.G. Lacko, A.K. Sood, Targeted delivery of small interfering RNA using reconstituted high-density lipoprotein nanoparticles, Neoplasia, 13 (2011) 309-U142.

[159] K.N. Liadaki, T. Liu, S. Xu, B.Y. Ishida, P.N. Duchateaux, J.P. Krieger, J. Kane, M. Krieger, V.I. Zannis, Binding of high density lipoprotein (HDL) and discoidal reconstituted HDL to the HDL receptor scavenger receptor class B type I. Effect of lipid association and APOA-I mutations on receptor binding, J. Biol. Chem., 275 (2000) $21262-21271$.

[160] K.K. Ng, J.F. Lovell, G. Zheng, Lipoprotein-inspired nanoparticles for cancer theranostics, Acc. Chem. Res., 44 (2011) 1105-1113.

[161] O.C. Farokhzad, R. Langer, Impact of nanotechnology on drug delivery, ACS Nano, 3 (2009) 16-20.

[162] J. Wang, J. Jia, J. Liu, H. He, W. Zhang, Z. Li, Tumor targeting effects of a novel modified paclitaxel-loaded discoidal mimic high density lipoproteins, Drug Deliv., 20 (2013) 356-363. 
[163] J.J. Zhang M, Liu J, He H, Liu L., A novel modified paclitaxel-loaded discoidal recombinant high-density lipoproteins: Preparation, characterizations and in vivo evaluation., Asian J. Pharm. Sci., 8 (2013) 11-18.

[164] J. Jia, Y. Xiao, J. Liu, W. Zhang, H. He, L. Chen, M. Zhang, Preparation, characterizations, and in vitro metabolic processes of paclitaxel-loaded discoidal recombinant high-density lipoproteins, J. Pharm. Sci., 101 (2012) 2900-2908. [165] N. Sabnis, S. Pratap, I. Akopova, P.W. Bowman, A.G. Lacko, Pre-clinical evaluation of rHDL encapsulated retinoids for the treatment of neuroblastoma, Front. Pediatr., 1 (2013) 6.

[166] B. Wang, Y. Yuan, L. Han, L. Ye, X. Shi, M. Feng, Recombinant lipoproteins reinforce cytotoxicity of doxorubicin to hepatocellular carcinoma, J. Drug Target., 22 (2014) 76-85.

[167] Y. Yuan, W. Wang, B. Wang, H. Zhu, B. Zhang, M. Feng, Delivery of hydrophilic drug doxorubicin hydrochloride-targeted liver using apoAI as carrier, J. Drug Target., 21 (2013) $367-374$.

[168] K.H. Cho, Rapamycin in lipoproteins for enhanced delivery, Cell Cycle, 11 (2012) 4298.

[169] K.H. Cho, Enhanced delivery of rapamycin by V156K-apoA-I high-density lipoprotein inhibits cellular proatherogenic effects and senescence and promotes tissue regeneration, J. Gerontol. A Biol. Sci. Med. Sci., 66 (2011) 1274-1285. [170] J. Tang, M.E. Lobatto, L. Hassing, S. van der Staay, S.M. van Rijs, C. Calcagno, M.S. Braza, S. Baxter, F. Fay, B.L. Sanchez-Gaytan, R. Duivenvoorden, H.B. Sager, Y.M. Astudillo, W. Leong, S. Ramachandran, G. Storm, C. Perez-Medina, T. Reiner, 
D.P. Cormode, G.J. Strijkers, E.S.G. Stroes, F.K. Swirski, M. Nahrendorf, E.A. Fisher, Z.A. Fayad, W.J.M. Mulder, Inhibiting macrophage proliferation suppresses atherosclerotic plaque inflammation, Sci. Adv., 1 (2015) e1400223.

[171] I.R. Corbin, Ligand-coupled lipoprotein for ovarian cancer-specific drug delivery, Methods Mol. Biol., 1049 (2013) 467-480.

[172] G.I. Harisa, F.K. Alanazi, Low density lipoprotein bionanoparticles: From cholesterol transport to delivery of anti-cancer drugs, Saudi Pharm. J., 22 (2014) 504515.

[173] A.N. Makheja, S. Bloom, R. Muesing, T. Simon, J.M. Bailey, Anti-inflammatory drugs in experimental atherosclerosis. 7. Spontaneous atherosclerosis in WHHL rabbits and inhibition by cortisone acetate, Atherosclerosis, 76 (1989) 155-161.

[174] M. Naito, M. Yasue, K. Asai, K. Yamada, T. Hayashi, M. Kuzuya, C. Funaki, N. Yoshimine, F. Kuzuya, Effects of dexamethasone on experimental atherosclerosis in cholesterol-fed rabbits, J. Nutr. Sci. Vitaminol. (Tokyo), 38 (1992) 255-264.

[175] K. Asai, C. Funaki, T. Hayashi, K. Yamada, M. Naito, M. Kuzuya, F. Yoshida, N. Yoshimine, F. Kuzuya, Dexamethasone-induced suppression of aortic atherosclerosis in cholesterol-fed rabbits. Possible mechanisms, Arterioscler. Thromb., 13 (1993) 892-899. [176] Y. Tauchi, M. Takase, I. Zushida, S. Chono, J. Sato, K. Ito, K. Morimoto, Preparation of a complex of dexamethasone palmitate-low density lipoprotein and its effect on foam cell formation of murine peritoneal macrophages, J. Pharm. Sci., 88 (1999) 709-714. 
[177] Y. Tauchi, L. Zushida, S. Chono, J. Sato, K. Ito, K. Morimoto, Effect of dexamethasone palmitate-low density lipoprotein complex on cholesterol ester accumulation in aorta of atherogenic model mice, Biol. Pharm. Bull., 24 (2001) 925-929. [178] A.C. Chu, S.Y. Tsang, E.H. Lo, K.P. Fung, Low density lipoprotein as a targeted carrier for doxorubicin in nude mice bearing human hepatoma HepG2 cells, Life Sci., 70 (2001) 591-601.

[179] M. Nikanjam, A.R. Gibbs, C.A. Hunt, T.F. Budinger, T.M. Forte, Synthetic nanoLDL with paclitaxel oleate as a targeted drug delivery vehicle for glioblastoma multiforme, J. Control. Release, 124 (2007) 163-171.

[180] A.A. Radwan, F.K. Alanazi, Design and synthesis of new cholesterol-conjugated 5Fluorouracil: a novel potential delivery system for cancer treatment, Molecules, 19 (2014) $13177-13187$.

[181] F. Meng, S. Asghar, S. Gao, Z. Su, J. Song, M. Huo, W. Meng, Q. Ping, Y. Xiao, A novel LDL-mimic nanocarrier for the targeted delivery of curcumin into the brain to treat Alzheimer's disease, Colloids Surf. B Biointerfaces, 134 (2015) 88-97.

[182] K.G. Parhofer, P. Hugh, R. Barrett, D.M. Bier, G. Schonfeld, Determination of kinetic parameters of apolipoprotein B metabolism using amino acids labeled with stable isotopes, J. Lipid Res., 32 (1991) 1311-1323.

[183] A. Kader, A. Pater, Loading anticancer drugs into HDL as well as LDL has little affect on properties of complexes and enhances cytotoxicity to human carcinoma cells, J. Control. Release, 80 (2002) 29-44.

[184] S.M. Grundy, H.Y. Mok, Chylomicron clearance in normal and hyperlipidemic man, Metabolism, 25 (1976) 1225-1239. 
[185] E.J. Schaefer, L.L. Jenkins, H.B. Brewer, Jr., Human chylomicron apolipoprotein metabolism, Biochem. Biophys. Res. Commun., 80 (1978) 405-412.

[186] J. Ahmad, S. Amin, M. Rahman, R.A. Rub, M. Singhal, M.Z. Ahmad, Z. Rahman, R.T. Addo, F.J. Ahmad, G. Mushtaq, M.A. Kamal, S. Akhter, Solid matrix based lipidic nanoparticles in oral cancer chemotherapy: applications and pharmacokinetics, Curr. Drug Metab., 16 (2015) 633-644.

[187] S. Han, L. Hu, T. Quach, J.S. Simpson, N.L. Trevaskis, C.J. Porter, Constitutive triglyceride turnover into the mesenteric lymph is unable to support efficient lymphatic transport of a biomimetic triglyceride prodrug, J. Pharm. Sci., (2015).

[188] V. Makwana, R. Jain, K. Patel, M. Nivsarkar, A. Joshi, Solid lipid nanoparticles (SLN) of Efavirenz as lymph targeting drug delivery system: Elucidation of mechanism of uptake using chylomicron flow blocking approach, Int. J. Pharm., 495 (2015) 439-446. [189] M.R. Aji Alex, A.J. Chacko, S. Jose, E.B. Souto, Lopinavir loaded solid lipid nanoparticles (SLN) for intestinal lymphatic targeting, Eur. J. Pharm. Sci., 42 (2011) 1118.

[190] P. Guo, O. Coban, N.M. Snead, J. Trebley, S. Hoeprich, S. Guo, Y. Shu, Engineering RNA for targeted siRNA delivery and medical application, Adv. Drug Deliv. Rev., 62 (2010) 650-666.

[191] T.M. Rana, Illuminating the silence: understanding the structure and function of small RNAs, Nat. Rev. Mol. Cell Biol., 8 (2007) 23-36.

[192] S. Weinstein, D. Peer, RNAi nanomedicines: challenges and opportunities within the immune system, Nanotechnology, 21 (2010) 232001. 
[193] K.M. McMahon, C.S. Thaxton, High-density lipoproteins for the systemic delivery of short interfering RNA, Expert Opin. Drug Deliv., 11 (2014) 231-247.

[194] Q. Chen, D. Butler, W. Querbes, R.K. Pandey, P. Ge, M.A. Maier, L. Zhang, K.G. Rajeev, L. Nechev, V. Kotelianski, M. Manoharan, D.W. Sah, Lipophilic siRNAs mediate efficient gene silencing in oligodendrocytes with direct CNS delivery, J. Control. Release, 144 (2010) 227-232.

[195] M. DiFiglia, M. Sena-Esteves, K. Chase, E. Sapp, E. Pfister, M. Sass, J. Yoder, P. Reeves, R.K. Pandey, K.G. Rajeev, M. Manoharan, D.W. Sah, P.D. Zamore, N. Aronin, Therapeutic silencing of mutant huntingtin with siRNA attenuates striatal and cortical neuropathology and behavioral deficits, Proc. Nat. Acad. Sci. USA, 104 (2007) 1720417209.

[196] J. Soutschek, A. Akinc, B. Bramlage, K. Charisse, R. Constien, M. Donoghue, S. Elbashir, A. Geick, P. Hadwiger, J. Harborth, M. John, V. Kesavan, G. Lavine, R.K. Pandey, T. Racie, K.G. Rajeev, I. Rohl, I. Toudjarska, G. Wang, S. Wuschko, D. Bumcrot, V. Koteliansky, S. Limmer, M. Manoharan, H.P. Vornlocher, Therapeutic silencing of an endogenous gene by systemic administration of modified siRNAs, Nature, 432 (2004) 173-178.

[197] C. Wolfrum, S. Shi, K.N. Jayaprakash, M. Jayaraman, G. Wang, R.K. Pandey, K.G. Rajeev, T. Nakayama, K. Charrise, E.M. Ndungo, T. Zimmermann, V. Koteliansky, M. Manoharan, M. Stoffel, Mechanisms and optimization of in vivo delivery of lipophilic siRNAs, Nat. Biotechnol., 25 (2007) 1149-1157.

[198] M.K. Bijsterbosch, E.T. Rump, R.L. De Vrueh, R. Dorland, R. van Veghel, K.L. Tivel, E.A. Biessen, T.J. van Berkel, M. Manoharan, Modulation of plasma protein 
binding and in vivo liver cell uptake of phosphorothioate oligodeoxynucleotides by cholesterol conjugation, Nucleic Acids Res., 28 (2000) 2717-2725.

[199] C. Lorenz, P. Hadwiger, M. John, H.P. Vornlocher, C. Unverzagt, Steroid and lipid conjugates of siRNAs to enhance cellular uptake and gene silencing in liver cells, Bioorg. Med. Chem. Lett., 14 (2004) 4975-4977.

[200] J. Wagner, M. Riwanto, C. Besler, A. Knau, S. Fichtlscherer, T. Roxe, A.M. Zeiher, U. Landmesser, S. Dimmeler, Characterization of levels and cellular transfer of circulating lipoprotein-bound microRNAs, Arterioscler. Thromb. Vasc. Biol., 33 (2013) 1392-1400.

[201] S. Yang, M.G. Damiano, H. Zhang, S. Tripathy, A.J. Luthi, J.S. Rink, A.V. Ugolkov, A.T. Singh, S.S. Dave, L.I. Gordon, C.S. Thaxton, Biomimetic, synthetic HDL nanostructures for lymphoma, Proc. Nat. Acad. Sci. USA, 110 (2013) 2511-2516. [202] Y. Ding, W. Wang, M. Feng, Y. Wang, J. Zhou, X. Ding, X. Zhou, C. Liu, R. Wang, Q. Zhang, A biomimetic nanovector-mediated targeted cholesterol-conjugated siRNA delivery for tumor gene therapy, Biomaterials, 33 (2012) 8893-8905. [203] M. Ghosh, G. Ren, J.B. Simonsen, R.O. Ryan, Cationic lipid nanodisks as an siRNA delivery vehicle, Biochem. Cell Biol., 92 (2014) 200-205.

[204] C.S. Thaxton, W.L. Daniel, D.A. Giljohann, A.D. Thomas, C.A. Mirkin, Templated spherical high density lipoprotein nanoparticles, J. Am. Chem. Soc., 131 (2009) 13841385.

[205] A.J. Luthi, N.N. Lyssenko, D. Quach, K.M. McMahon, J.S. Millar, K.C. Vickers, D.J. Rader, M. Phillips, C.A. Mirkin, C.S. Thaxton, Robust passive and active efflux of 
cellular cholesterol to a designer functional mimic of high density lipoprotein, J. Lipid Res., 56 (2015) 972-985.

[206] S. Tripathy, E. Vinokour, K.M. McMahon, O.V. Volpert, C.S. Thaxton, High density lipoprotein nanoparticles deliver RNAi to endothelial cells to inhibit angiogenesis, Part. Part. Syst. Charact., 31 (2014) 1141-1150.

[207] M. Yang, H. Jin, J. Chen, L. Ding, K.K. Ng, Q. Lin, J.F. Lovell, Z. Zhang, G. Zheng, Efficient cytosolic delivery of siRNA using HDL-mimicking nanoparticles, Small, 7 (2011) 568-573.

[208] H. Jin, J.F. Lovell, J. Chen, Q. Lin, L. Ding, K.K. Ng, R.K. Pandey, M. Manoharan, Z. Zhang, G. Zheng, Mechanistic insights into LDL nanoparticle-mediated siRNA delivery, Bioconjugate Chem, 23 (2012) 33-41.

[209] Q.L. Zhu, Y. Zhou, M. Guan, X.F. Zhou, S.D. Yang, Y. Liu, W.L. Chen, C.G. Zhang, Z.Q. Yuan, C. Liu, A.J. Zhu, X.N. Zhang, Low-density lipoprotein-coupled Nsuccinyl chitosan nanoparticles co-delivering siRNA and doxorubicin for hepatocytetargeted therapy, Biomaterials, 35 (2014) 5965-5976.

[210] H. Kuwahara, K. Nishina, K. Yoshida, T. Nishina, M. Yamamoto, Y. Saito, W. Piao, M. Yoshida, H. Mizusawa, T. Yokota, Efficient in vivo delivery of siRNA into brain capillary endothelial cells along with endogenous lipoprotein, Mol. Ther., 19 (2011) 2213-2221.

[211] P. Boggio, A. Toppino, S. Geninatti Crich, D. Alberti, D. Marabello, C. Medana, C. Prandi, P. Venturello, S. Aime, A. Deagostino, The hydroboration reaction as a key for a straightforward synthesis of new MRI-NCT agents, Org. Biomol. Chem., 13 (2015) 3288-3297. 
[212] D. Alberti, N. Protti, A. Toppino, A. Deagostino, S. Lanzardo, S. Bortolussi, S. Altieri, C. Voena, R. Chiarle, S. Geninatti Crich, S. Aime, A theranostic approach based on the use of a dual boron/Gd agent to improve the efficacy of Boron Neutron Capture Therapy in the lung cancer treatment, Nanomedicine (Lond), 11 (2015) 741-750.

[213] S. Geninatti-Crich, D. Alberti, I. Szabo, A. Deagostino, A. Toppino, A. Barge, F. Ballarini, S. Bortolussi, P. Bruschi, N. Protti, S. Stella, S. Altieri, P. Venturello, S. Aime, MRI-guided neutron capture therapy by use of a dual gadolinium/boron agent targeted at tumour cells through upregulated low-density lipoprotein transporters, Chem. Eur. J., 17 (2011) 8479-8486.

[214] A. Toppino, M.E. Bova, S. Geninatti Crich, D. Alberti, E. Diana, A. Barge, S. Aime, P. Venturello, A. Deagostino, A carborane-derivative "click" reaction under heterogeneous conditions for the synthesis of a promising lipophilic MRI/GdBNCT agent, Chem. Eur. J., 19 (2013) 721-728.

[215] R.F. Barth, M.G.H. Vicente, O.K. Harling, W.S. Kiger, K.J. Riley, P.J. Binns, F.M. Wagner, M. Suzuki, T. Aihara, I. Kato, S. Kawabata, Current status of boron neutron capture therapy of high grade gliomas and recurrent head and neck cancer, Radiat. Oncol., 7 (2012) 146.

[216] R.S. Kootte, L.P. Smits, F.M. van der Valk, J.-L. Dasseux, C.H. Keyserling, R. Barbaras, J.F. Paolini, R.D. Santos, T.H. van Dijk, G.M. Dallinga-van Thie, A.J. Nederveen, W.J.M. Mulder, G.K. Hovingh, J.J.P. Kastelein, A.K. Groen, E.S. Stroes, Effect of open-label infusion of an apoA-I-containing particle (CER-001) on RCT and artery wall thickness in patients with FHA, J. Lipid Res., 56 (2015) 703-712. 
[217] M.A. Sieber, H. Pietsch, J. Walter, W. Haider, T. Frenzel, H.J. Weinmann, A preclinical study to investigate the development of nephrogenic systemic fibrosis: A possible role for gadolinium-based contrast media, Investigative Radiol., 43 (2008) 6575.

[218] E. Kanal, M.F. Tweedle, Residual or retained gadolinium: practical implications for radiologists and our patients, Radiology, 275 (2015) 630-634. 


\section{Figure Legends}

Figure 1 Schematic depiction of the different lipoproteins, indicating their different compositions (not to scale). Figure reproduced with permission from reference [50].

Figure 2 Illustration of the numerous ways in which lipoproteins can be modified to act as contrast agents, therapeutics, or both. Multiple of these modifications can be made on the same platform, e.g. inclusion of a targeting ligand and a fluorophore.[43]

Figure 3 HDL-based nanoparticle active for PET, NIRF and photodynamic therapy. A) Schematic depiction of the HDL-based nanoparticle. B) Negative stain TEM characterization of the nanoparticles (scale bars are 100 and $20 \mathrm{~nm}$ in the larger view and inset, respectively). C) Analysis of photodynamic therapy effects on glioma tumors (PLP is the term for the nanoparticle). D) PET imaging of prostate cancer. E) Fluorescence molecular tomography of prostate cancer. F) Interoperative imaging aiding prostate cancer resection i) before and ii) after resection. Figure reproduced with permission from reference [120].

Figure 4 Gold-core LDL (Au-LDL) as a contrast agent for computed tomography. A) Schematic depiction of Au-LDL. B-D) Negative stain TEM images of lipid-coated gold nanoparticles (Au-MHPC), LDL and Au-LDL. E-G) Fluorescence microscopy images of B16-F10 cells that have been incubated with various agents. H) Gold content of blood of wild type (WT) and LDL receptor knockout (LDLr KO) mice injected with Au-LDL. I, J) Spectral CT images of a mouse bearing a B16-F10 tumor. Bones are denoted in grayscale 
and gold is given a yellow color. This figure is reproduced with permission from reference [39].

Figure 5 Statin loaded HDL as a therapeutic in atherosclerosis. A) Schematic depictions of statin loaded HDL, as well as TEM characterization. B) MR imaging of the aorta of an atherosclerotic mouse pre- and 24 hours post-injection with [Gd-dye-S]-rHDL. C) Fluorescence imaging of the excised aortas of atherosclerotic mice that had been injected with either saline or [Gd-dye-S]-rHDL 24 hours previously. D) Histology of the aorta roots of atherosclerotic mice. E,F) Quantitative analysis of histology images. Figure reproduced with permission from reference [44].

Figure 6 Gold core HDL for DNA delivery. A) Schematic depiction of gold core HDL. B) Results from an LDH assay showing that the formulation is biocompatible. C) Suppression of miR-210 by DNA-HDL conjugates. D) Western blot of E2F3a (an miR210 target) levels. Figure reproduced with permission from reference [92]. 
Apolipoprotein $\begin{array}{cccc}\mathrm{CH} & \mathrm{CE} & \mathrm{TG} & \mathrm{PL}\end{array}$

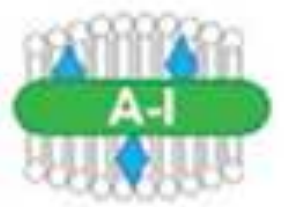

\section{Nascent $\mathrm{HDL}$}

\section{A-1}

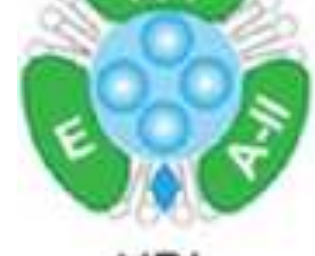

HDL
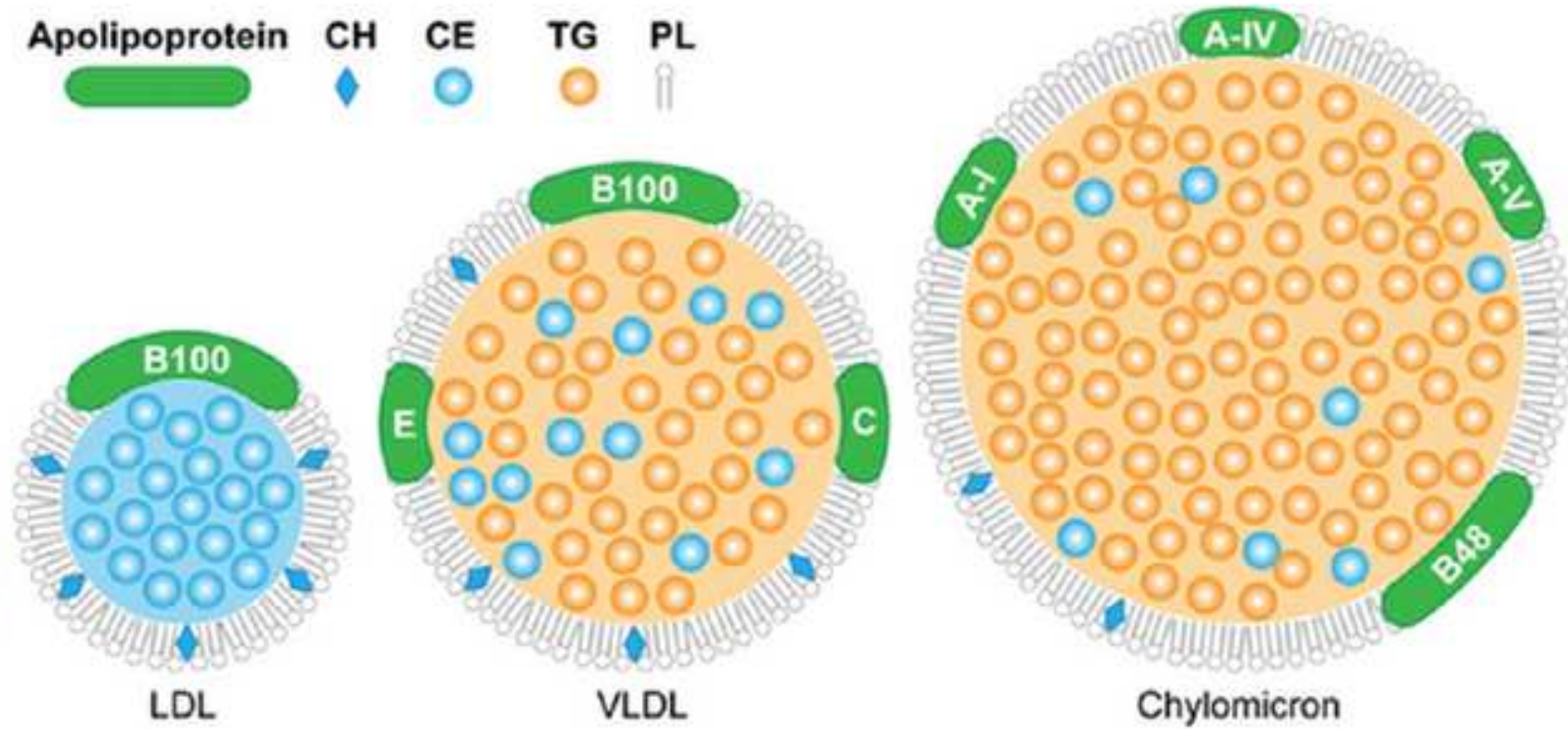


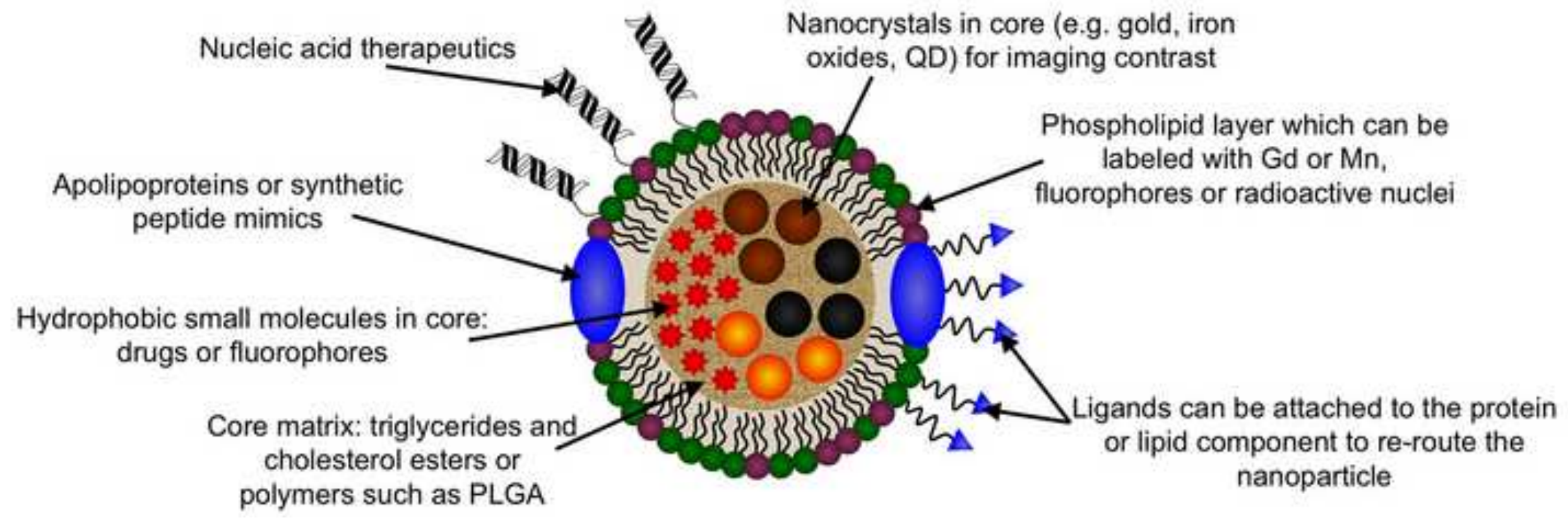



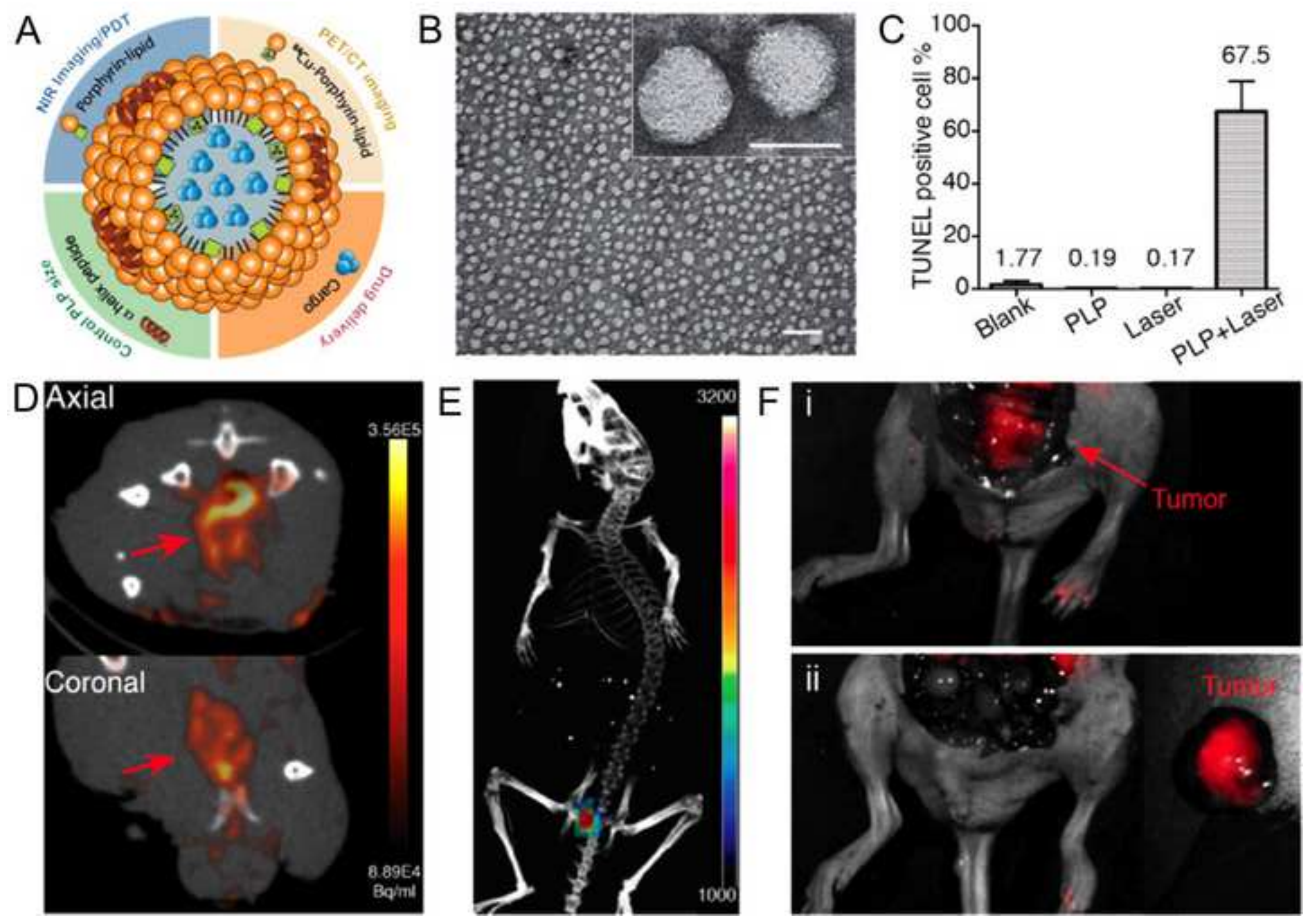

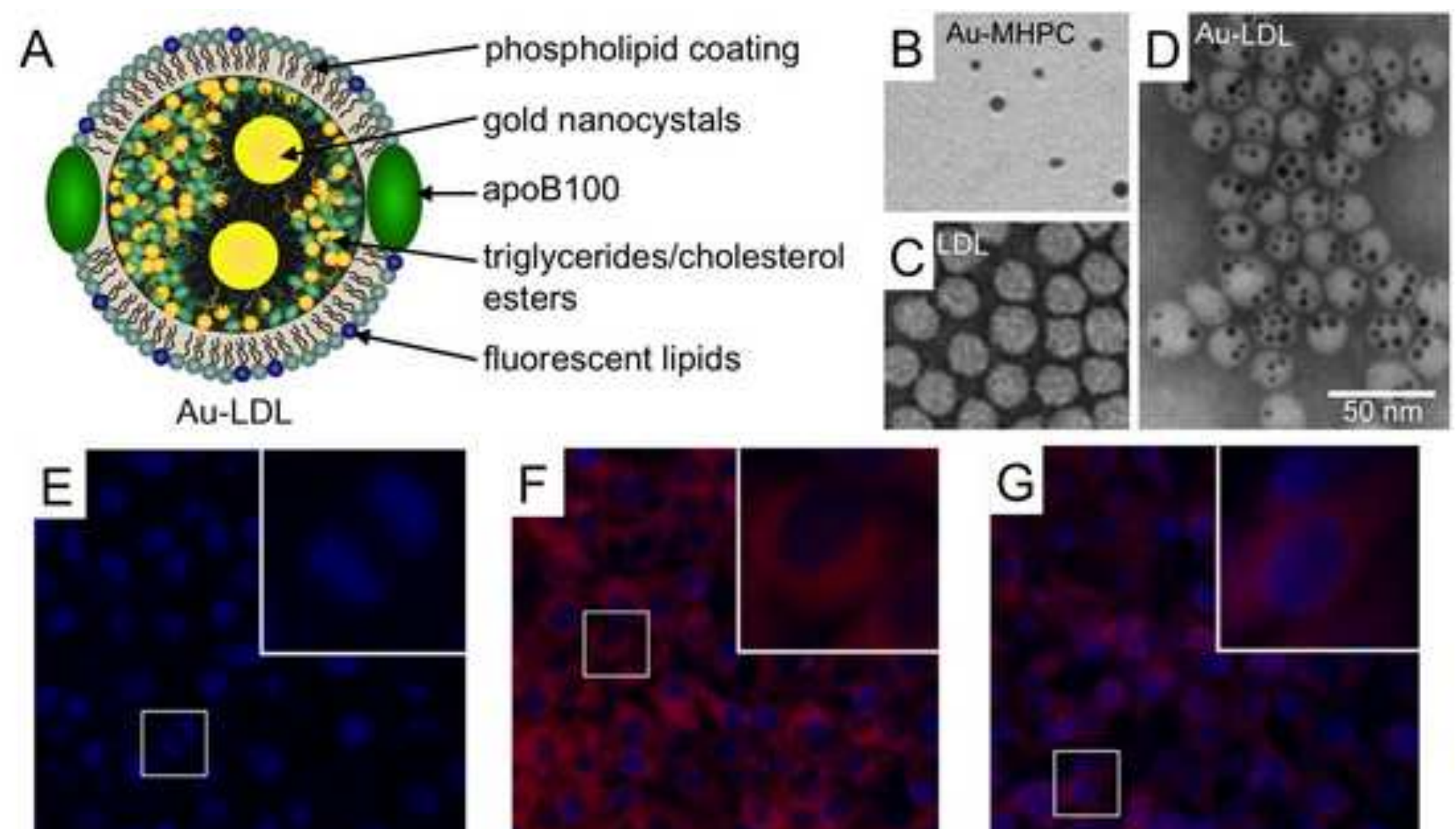

\section{B16-F10: LDL}
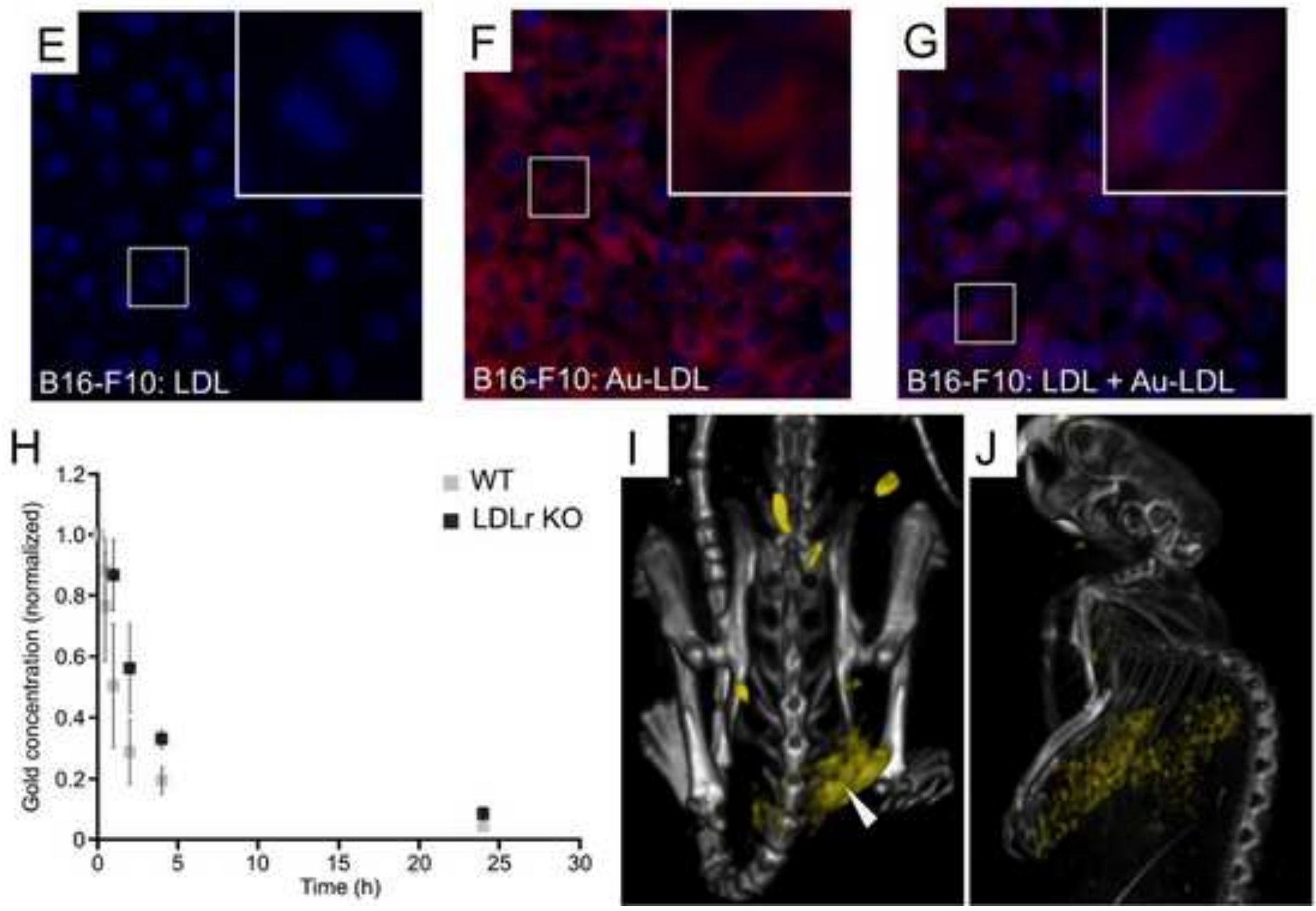

B16-F10: Au-LDL

$B 16-F 10: L D L+A u-L D L$ 


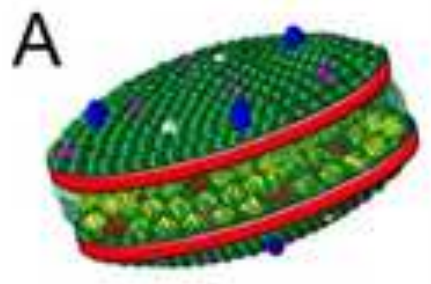

[Gd-dye-S]-rHDL

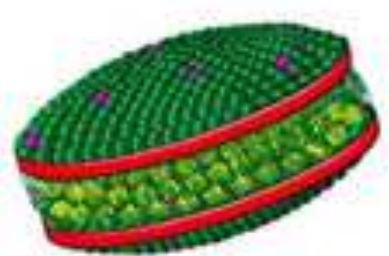

[S]-rHDL
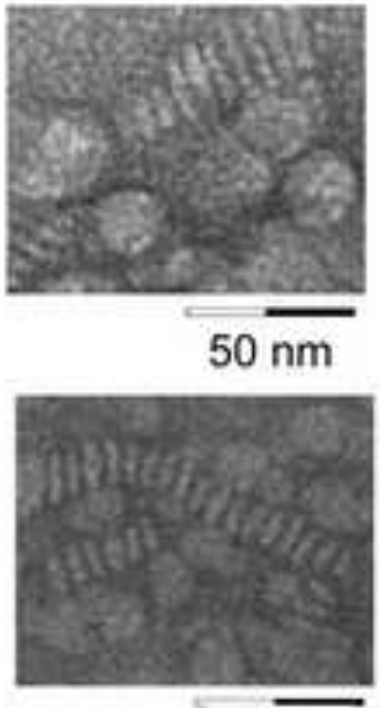

$50 \mathrm{~nm}$
- $\sim 0$

ApoA1 MHPC DMPE Cy5.5-DMPE

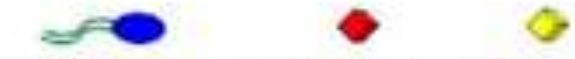

Gd-DTPA- DiO/DiR Simvastatin DMPE
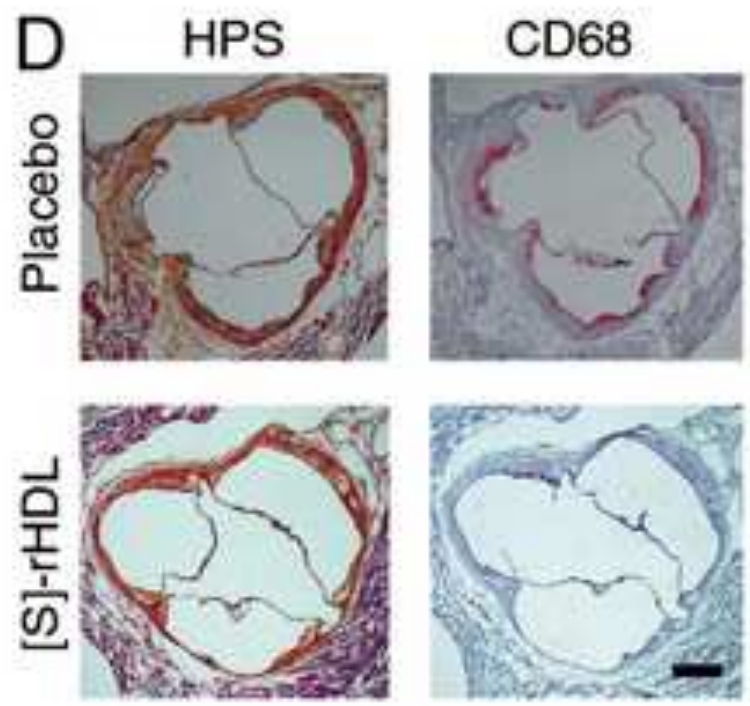

B

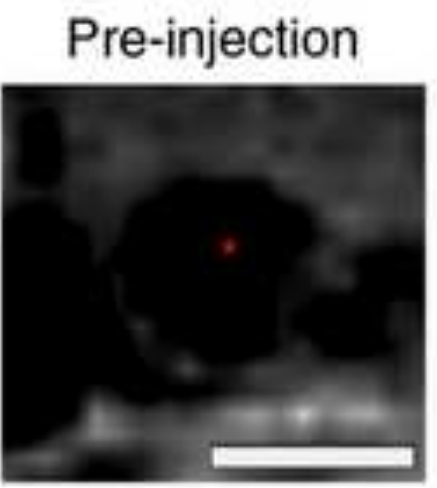

$24 \mathrm{~h}$ post-injection

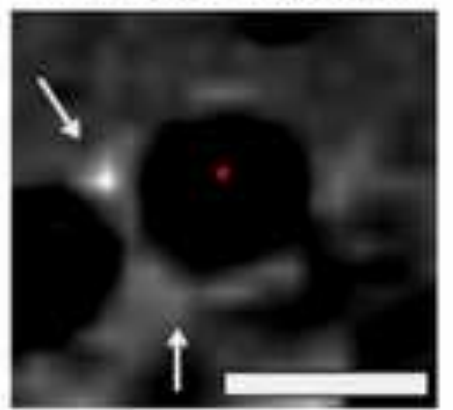

C
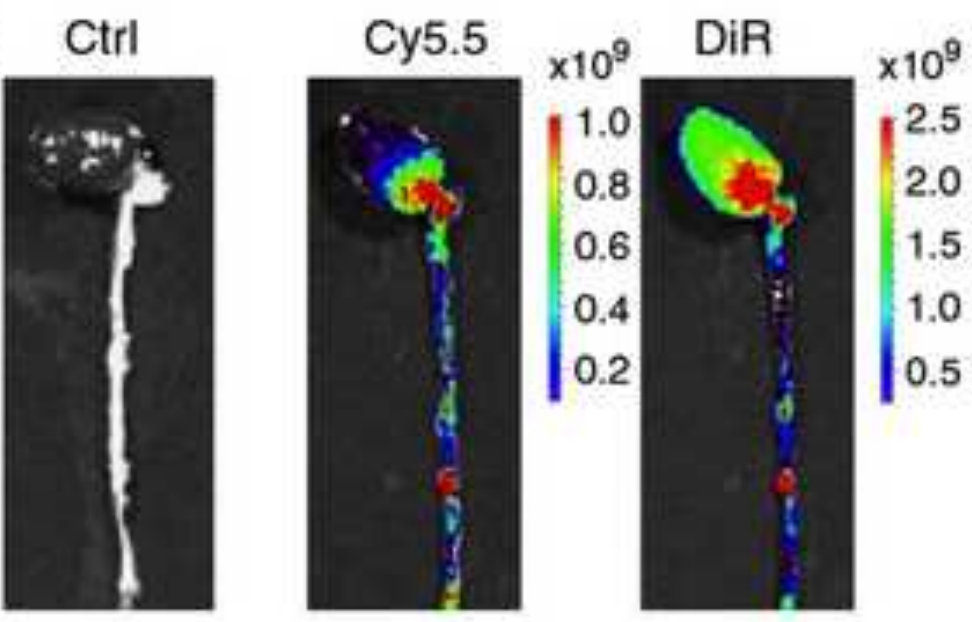

E
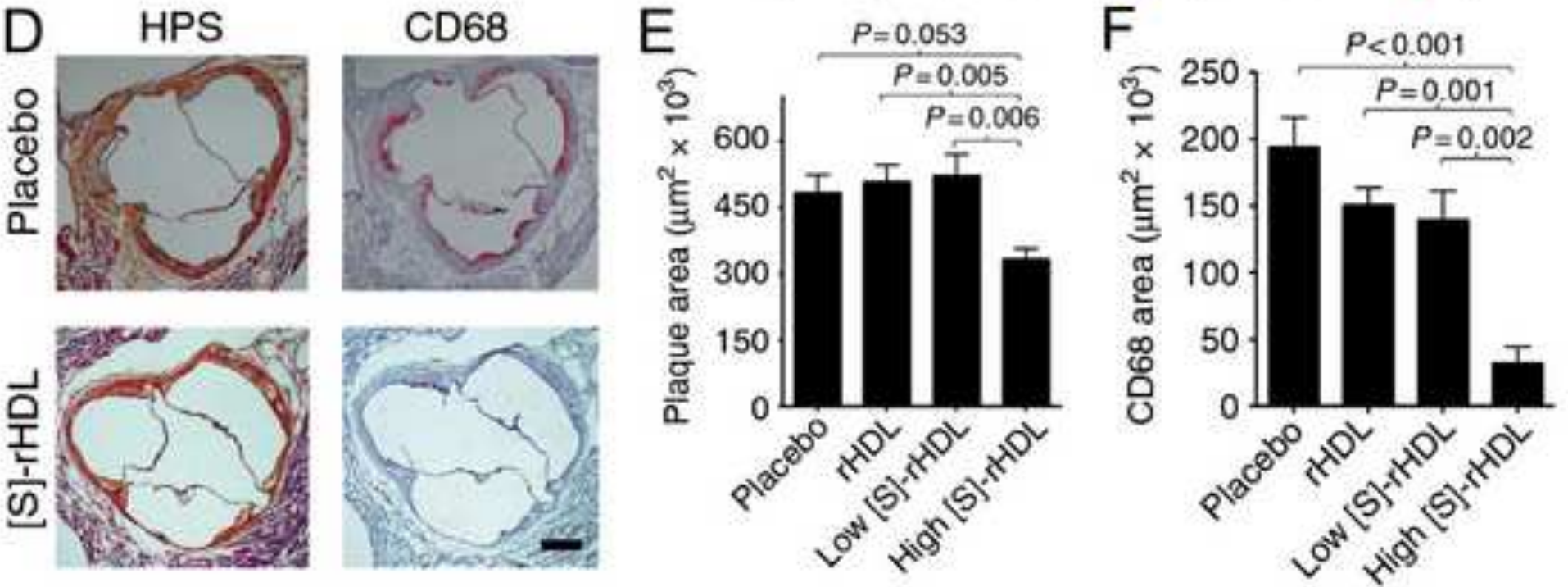

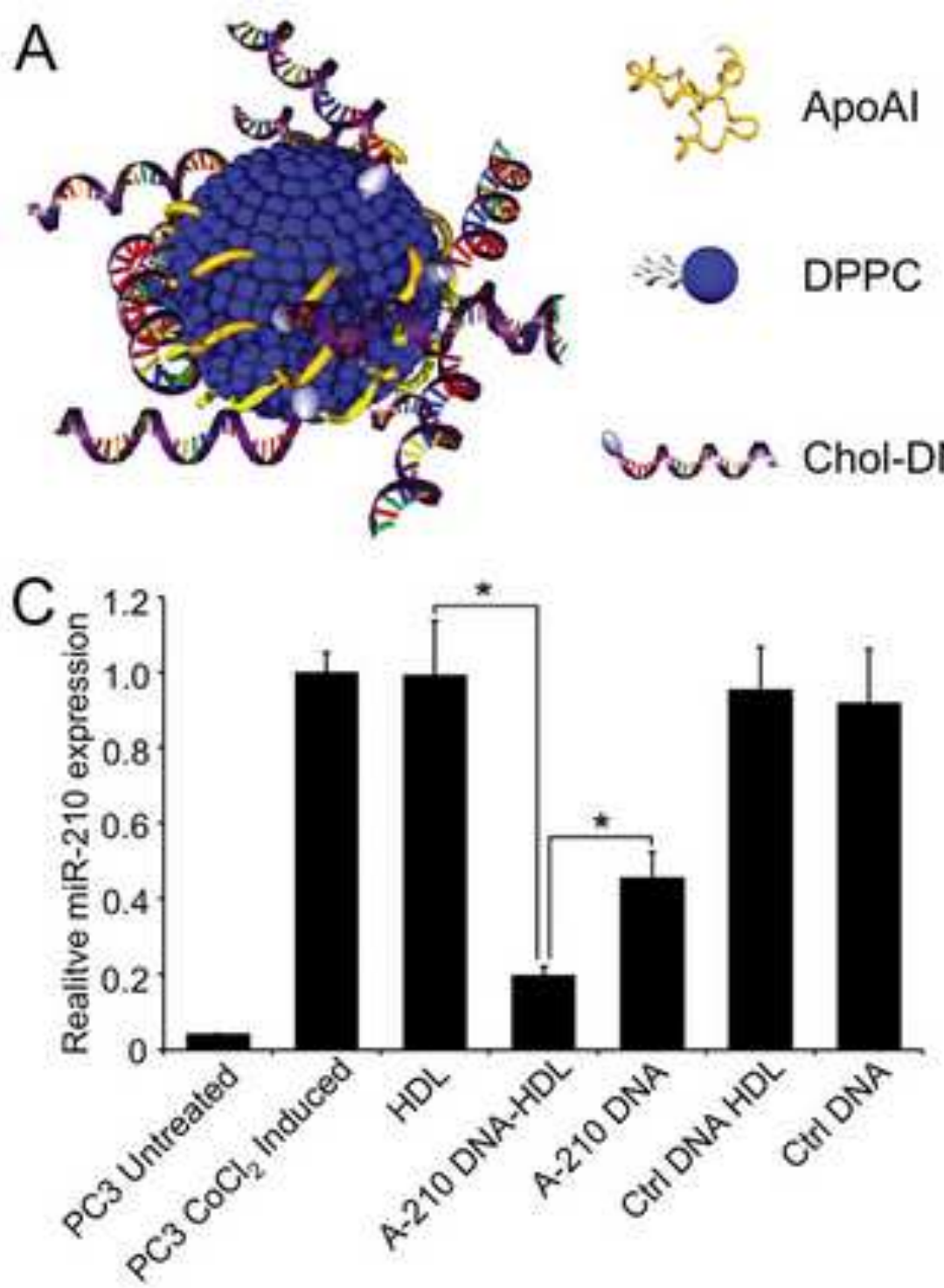

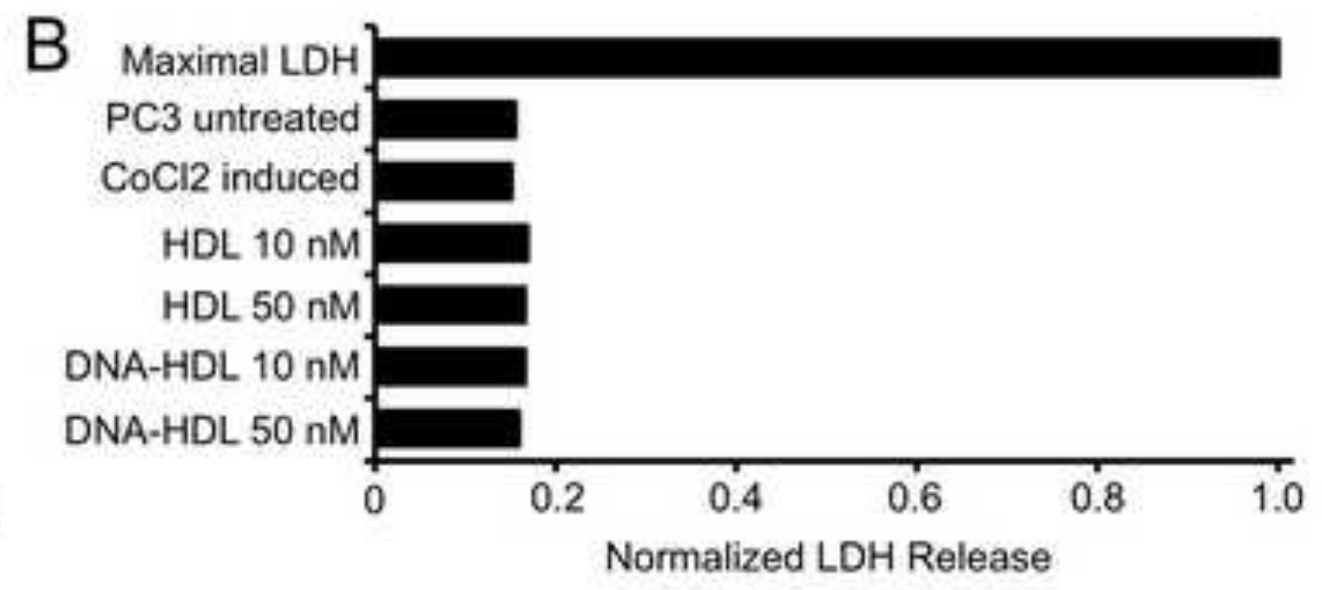

D

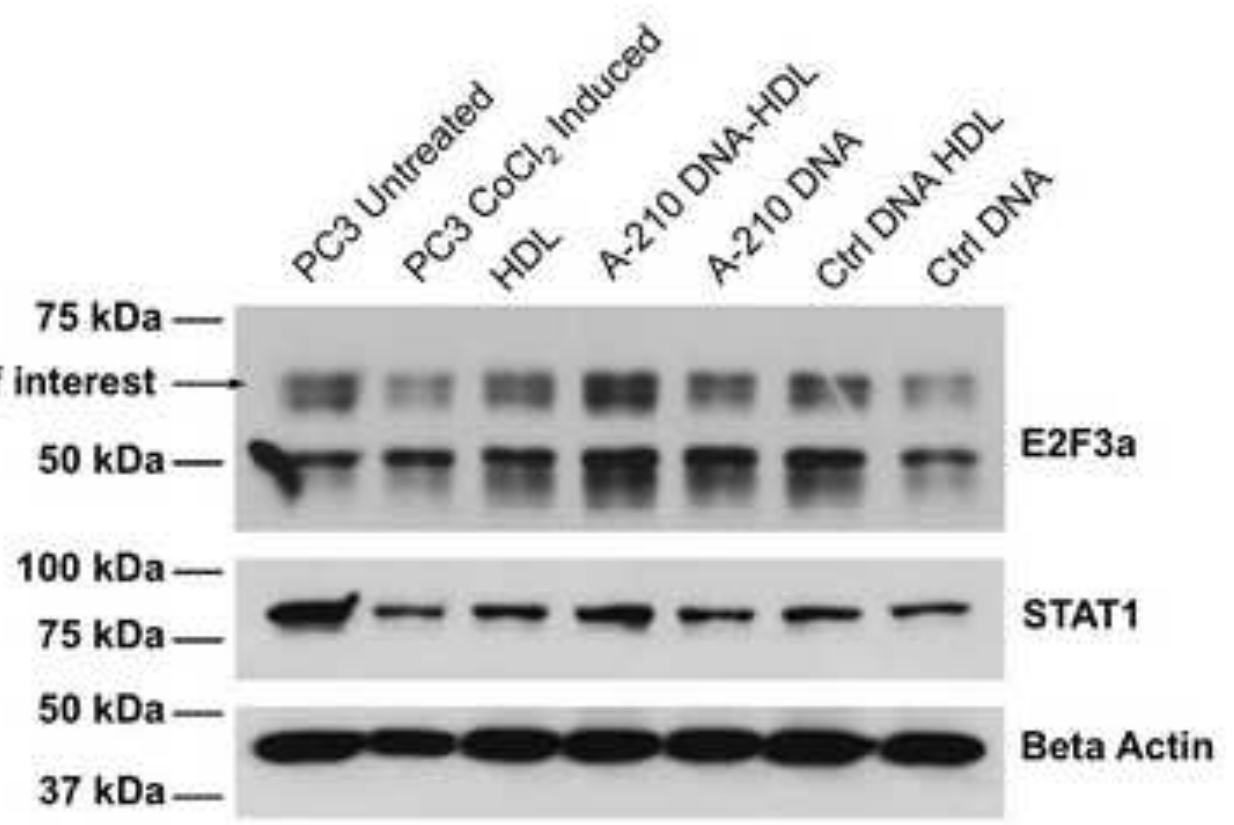




\begin{tabular}{|c|c|c|c|c|}
\hline Lipoprotein & Size (nm) & $\begin{array}{c}\text { Major } \\
\text { apolipoprotein }\end{array}$ & Density (g/dl) & Major role \\
\hline Chylomicron & $80-1200$ & $\begin{array}{c}\text { B-48, A-I, A- } \\
\text { II, C, E }\end{array}$ & $<0.95$ & Transport of dietary fats \\
\hline VLDL & $35-80$ & B-100, C, E & $0.95-1.006$ & $\begin{array}{c}\text { Transport of fats from the } \\
\text { liver }\end{array}$ \\
\hline IDL & $27-30$ & B-100, C, E & $1.006-1.019$ & $\begin{array}{c}\text { Transport of fats from the } \\
\text { liver }\end{array}$ \\
\hline LDL & $22-27$ & B-100 & $1.019-1.063$ & $\begin{array}{c}\text { Delivery of fats to } \\
\text { peripheral tissues }\end{array}$ \\
\hline HDL & $7-13$ & A-I, A-II, C, E & $1.063-1.25$ & $\begin{array}{c}\text { Removal of cholesterol } \\
\text { from peripheral tissues }\end{array}$ \\
\hline
\end{tabular}

Table 1. Summary of lipoprotein characteristics. Data sourced from [48]. 


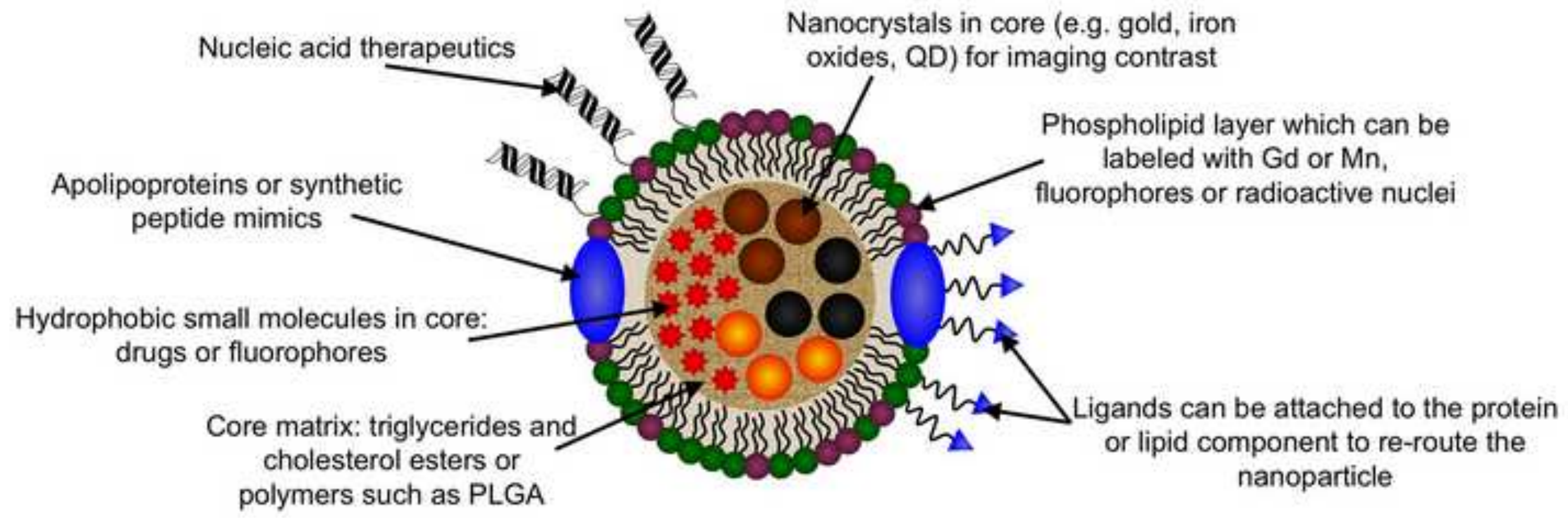

